\title{
Information disclosure by a seller in sequential first-price auctions
}

\section{Helmuts Āzacis ${ }^{1}$}

Accepted: 16 January 2020 / Published online: 27 January 2020

(c) The Author(s) 2020

\begin{abstract}
I study sequential first-price auctions where two items are sold to two bidders with private binary valuations. A seller, prior to the second auction, can publicly disclose some information about the outcome of the first auction. I characterize equilibrium strategies for various disclosure rules when the valuations of bidders are either perfectly positively or perfectly negatively correlated across items. I establish outcome equivalence between different disclosure rules. I find that it is optimal for the seller to disclose some information when the valuations are negatively correlated, whereas it is optimal not to disclose any information when the valuations are positively correlated. For most of the parameter values, the seller's expected revenue is higher if the losing bid is disclosed. When only the winner's identity is disclosed, the equilibrium is efficient whether the valuations are positively or negatively correlated.
\end{abstract}

Keywords Efficiency $\cdot$ Information disclosure $\cdot$ Seller's revenue $\cdot$ Sequential first-price auctions

JEL Classification D44 · D47 · D82

\section{Introduction}

Auction houses like Sotheby's and Christie's usually sell multiple items in a sequence on the same auction day. At the Aalsmeer Flower Auction in the Netherlands, many

\footnotetext{
I would like to thank the associated editor, the referee, Gueorgui Kolev, Deniz Okat, Thomas Tröger, Péter Vida, the seminar participants at Brunel University London, Paris Dauphine University, Queen's University Belfast, and the audiences at the 2013 Asian Meeting of the Econometric Society, the 9th Spain-Italy-The Netherlands Meeting on Game Theory, the 6th Annual Lithuanian Conference on Economic Research, and the 2017 Annual Meeting of the Association of Southern European Economic Theorists for their valuable comments.
}

$凶$ Helmuts Āzacis AzacisH@cf.ac.uk

1 Cardiff Business School, Cardiff University, Aberconway Building, Colum Drive, Cardiff CF10 3EU, Wales, UK 
lots of flowers are sold continuously each day. Governments across the world procure goods and services through auctions. Even though a single procurement contract might be awarded in a particular auction, these auctions are often recurring events with the same set of bidders. Because of the sequential nature of auctions in these examples, the auctioneers have a choice how much information to disclose about the outcomes of earlier auctions prior to the start of next auction.

I study this question in a setup where an auctioneer sells two items sequentially using first-price auctions to two bidders, whose valuations for each item can take one of two possible values. I consider the following disclosure rules by the seller: (1) disclose both the winning and losing bids of the first auction, (2) only the winning bid, (3) only the losing bid, (4) only the winner's identity, or (5) do not disclose anything. The objective is to identify among these disclosure rules the one that maximizes the seller's expected revenue. ${ }^{12}$

Although the bidders' valuations of the items are assumed to be independent across bidders, they are correlated across items. Specifically, I consider two extreme cases. The first case considered is when the item valuations are perfectly negatively correlated, meaning, if a bidder has a high value for one item, then he has a low value for the other item and vice versa. The second case considered is when the item valuations are perfectly positively correlated, meaning, a bidder has either high or low values for both items. ${ }^{3}$ By considering these two extreme cases, one can verify whether the optimal disclosure rule depends on the prior distribution of valuations.

The assumption of valuations being perfectly positively correlated is standard in the literature (to be discussed in Sect. 1.1). To justify the case of negative correlation, suppose two paintings, say, one by Rembrandt and the other by Picasso, are auctioned. One can imagine that each participating bidder has strong interest in one of the paintings because it would better complement his collection, but the other bidders are unsure which painting it is. Alternatively, the auctioned items can be physically located at different places (as is often the case in the auctions of natural resources like timber) and a bidder must incur transportation costs to collect them. If a bidder is located closer to one item than the other, then because of the transportation costs, he will attach higher value to the former item. Further, if other bidders are unsure about this bidder's location (for example, because the bidder's true identity is unknown), this creates uncertainty about his valuations.

I establish a set of results. First of all, I find equilibrium bidding strategies in the sequential first-price auctions for each combination of disclosure rule and the correlation of valuations (that is, ten different cases in total). Once the equilibrium strategies are found, I compare the seller's revenue from different disclosure rules to find the following. If the valuations of items are negatively correlated, then it is

\footnotetext{
${ }^{1}$ I only look for the optimal disclosure rule between the five rules that I consider, but I show in Sect. 5.4 that there can be even better disclosure rules.

${ }^{2}$ In the continuation, I will often refer to expected revenue simply as revenue. Since I only consider expected revenue, it should not cause confusion. The same comment also applies to surplus and bidder's payoff.

3 The case of perfectly negatively correlated valuations can be extended to more than two items by assuming that a bidder attaches a high value to exactly one item, while the case of perfectly positively correlated valuations can be extended by assuming that a bidder either attaches high values to all items or to none at all.
} 
beneficial for the seller to disclose some information, whereas if the valuations are positively correlated, then any information disclosure harms the seller.

The intuition for this result is as follows. If the seller discloses some information from the first auction, it allows the bidders to update their beliefs about the opponent. This, in turn, gives incentives to the bidders to conceal information about their valuations in the first auction. In particular, a bidder who has high value for the second item, benefits if he is perceived by the opponent as someone who has low value for that item because then the bidding in the second auction will be less aggressive.

The consequences from concealing their true valuations depend on the correlation of valuations across items. If the valuations are perfectly negatively correlated, then type $(0,1)$ bidder $^{4}$ tries to disguise himself as type $(1,0)$ bidder. Because the latter type bids aggressively for the first item, it means that type $(0,1)$ bidder must also bid relatively aggressively for that item even though his valuation of the item is low. In equilibrium, he even bids above his valuation of the first item with a positive probability. As a result, what type $(0,1)$ bidder gains in the second auction, he loses from more aggressive bidding in the first auction, so that his overall payoff is exactly the same that he would obtain if there was no information disclosure. ${ }^{5}$ In turn, type $(1,0)$ bidder is strictly worse off: he loses from more aggressive bidding in the first auction, but he does not benefit from less aggressive bidding in the second auction. Because this type loses, the seller gains although not necessarily to the same extent as some surplus can be lost due to inefficient allocation of items.

If the valuations are perfectly positively correlated, then type $(1,1)$ bidder tries to disguise himself as type $(0,0)$ bidder. Because the latter type does not bid aggressively for the first item, it means that type $(1,1)$ bidder also does not bid too aggressively for that item even though his valuation is high. ${ }^{6}$ Consequently, the seller loses from less aggressive bidding in both auctions and is better off by not disclosing any information.

I also establish outcome equivalence between various disclosure rules, which is summarized in Table 1. Interestingly, which rules are outcome equivalent again depends on the correlation between valuations. Thus, I find that disclosing the winning bid is equivalent to disclosing both bids if the valuations are negatively correlated, but it is equivalent to disclosing the winner's identity if the valuations are positively correlated. And it is opposite for the losing bid.

The intuition for these results can also be related back to the information updating between the first and second auctions and to the properties of the equilibrium in the second auction. At the start of the second auction, each bidder assigns some probability that the opponent has low value for the second item. Let's refer to the bidder who has higher probability of having low value as the weak bidder. Roughly, all that matters for the equilibrium payoffs in the second auction is the probability with which the

\footnotetext{
4 The type will be denoted by an ordered pair $(i, j) \in\{0,1\}^{2}$, where the first and second entries indicate bidder's valuations of the first and second items, respectively, while 0 stands for "low value" and 1 for "high value". Note, however, the high value will not necessarily be equal to 1 , although the low value will always be normalized to 0 .

5 His payoff would be strictly higher if the other type bid as he does when no information is disclosed. However, type $(1,0)$ bidder adjusts his strategy to more aggressive bidding by type $(0,1)$ bidder.

6 Bidding low in the first auction involves a cost to type $(1,1)$ bidder: the possibility of losing a high value item. Therefore, different types still bid differently in the equilibrium.
} 
Table 1 Equivalence and efficiency of disclosure rules

\begin{tabular}{lll}
\hline Disclosure rule & Correlation & \\
\cline { 2 - 3 } & Negative & Positive \\
\hline Winning bid & Both bids & Winner's identity \\
& Inefficient & Efficient \\
Losing bid & Winner's identity & Both bids \\
& Efficient & Inefficient
\end{tabular}

weak bidder has low value. Next, one can ask which of the two bidders-the winner or the loser of the first auction-is more likely to be the weak bidder.

When the valuations are negatively correlated, then between the types $(1,0)$ and $(0,1)$, it is likelier that the former type will win the first auction. Therefore, the winner of the first auction will be perceived as the weak bidder in the second auction. This intuitively explains why, on one hand, those disclosure rules that reveal the winner's bid are equivalent and, on the other hand, those disclosure rules that do not reveal the winner's bid are equivalent. When the valuations are positively correlated, then between the types $(0,0)$ and $(1,1)$, it is likelier that the latter type will win the first auction. Therefore, the loser of the first auction will be perceived as the weak bidder in the second auction. This again explains why, on one hand, those disclosure rules that reveal the loser's bid are equivalent and, on the other hand, those disclosure rules that do not reveal the loser's bid are equivalent.

One can also be more specific on the revenue ranking of the disclosure rules. Given the equivalence between different disclosure rules, it is enough to compare the revenues from disclosing the winning bid against disclosing the losing bid. I find that irrespective of the sign of correlation, for most of the parameter values, the bidders bid more aggressively when the losing bid is announced, leading to lower payoffs to the bidders and higher revenue to the seller. Though, as argued before, if the valuations are positively correlated, then any information disclosure is dominated by no disclosure.

There also exists a close relationship between the disclosure rule and the efficiency of the equilibrium outcome in the first auction. ${ }^{7}$ I find that the equilibrium is inefficient (resp., efficient) when the bid of the weak bidder is disclosed (resp., not disclosed). Therefore, if the objective of the seller is to implement an efficient outcome irrespective of the sign of correlation between the valuations of the items, she should only announce the winner's identity.

To understand this connection between the disclosure rule and efficiency, consider, for example, the case of negatively correlated valuations. Suppose there is an efficient and, consequently, separating equilibrium in the first auction and both bidders are of type $(0,1)$. When the winning bid (that is, the bid of the weak bidder) is disclosed, it becomes common knowledge that both bidders are of type $(0,1)$ and they bid aggressively in the second auction. To avoid it, type $(0,1)$ wants to pool with the other type and, as a result, the equilibrium is inefficient. On the other hand, when only the losing bid is disclosed, the loser still faces uncertainty about the type of the winner and

\footnotetext{
7 Because of the assumption of binary valuations, the equilibrium outcome of the second auction is always efficient.
} 
the bidding in the second auction is not so aggressive. This removes the need for type $(0,1)$ to pool with the other type. Similar intuition applies to the case with positively correlated valuations.

Finally, I check how robust the results are to departures from the benchmark model. Thus, I consider examples of imperfectly correlated valuations, asymmetric bidders, and more than two bidders. I find that most of the results continue to hold in the case of imperfectly correlated valuations, suggesting that the assumption of perfectly correlated valuations is not that restrictive. Also, when the bidders are asymmetric and the valuations are negatively correlated, the seller still benefits from disclosing the winning bid, but the equivalence between disclosing the winning bid and both bids does not hold anymore. Similarly, having more than two bidders changes the equivalence results between different disclosure rules. Thus, I find that with negatively correlated valuations, announcing the winning bid is now equivalent to not announcing anything. Finally, for the case of negatively correlated valuations, I also provide an example of more complicated disclosure rule that outperforms the disclosure rules considered so far. In particular, I assume that the seller announces the winning bid if it is below some threshold value, otherwise she announces the losing bid.

\subsection{Related literature}

I am not the first to study information disclosure in sequential auctions when bidders have multi-unit demand and they know their valuations for all items before any bidding takes place. ${ }^{8}$ To the best of my knowledge, however, all the existing studies assume that the valuations across items are perfectly positively correlated. I am the first to explore how the equilibrium strategies and the equilibrium outcomes depend on the sign of correlation between the valuations of the items.

The studies closest to this one are Thomas (2010), Cason et al. (2011), Kannan (2012) that also investigate the effects of information disclosure in sequential auctions' setup with two items and binary valuations. ${ }^{9}$ They all, however, assume that each bidder attaches the same value to both items. Apart from covering the case of negatively correlated valuations, I also contribute to this literature when the valuations are perfectly positively correlated, by allowing the high values of the two items to differ. Thus, for some priors, I find that the ranking of revenues from different disclosure rules depends on the relative sizes of the high values for both items. Additionally, I consider a wider range of disclosure rules.

There are also other studies that use the same setup as the aforementioned studies to explore issues other than information disclosure. Thus, Ding et al. (2010) study the dynamics of equilibrium prices when both bids are announced; Yao and Xiao (2013) compare the revenues from the simultaneous and sequential auctions when only the winning bid is announced in the latter auctions; Âzacis and Vida (2012) illustrate

\footnotetext{
8 Jeitschko (1998) shows that even in the unit demand case, announcing the winning bid affects bidders' beliefs and, hence, their equilibrium strategies when they have discrete valuations. However, the equilibrium revenues in the sequential and simultaneous auctions are still the same.

9 All these studies consider procurement auctions, but it is inconsequential for the results.
} 
how the announcement of the winning bid naturally leads to the information exchange between the bidders about their valuations.

Almost all studies that analyse the effects of information disclosure in sequential auctions with multi-unit demand assume binary valuations. Two exceptions are Février (2003) and Tu (2005), which assume that the valuations are drawn from a continuous distribution. Similar to my results, Février (2003) finds that when the valuations are positively correlated, the seller is better off by not disclosing any information than announcing the winner's identity. Tu (2005), similar to this study, considers a range of disclosure rules, but additionally he requires the equilibrium strategies to be monotonically increasing in valuations. This requirement rules out pooling equilibria and even leads to the non-existence of equilibrium for some disclosure rules.

All studies that are cited above, assume a sequence of two auctions. Bergemann and Hörner (2017) depart from this assumption and instead consider an infinite sequence of auctions, but still assume binary and constant valuations. To rule out explicit collusion by bidders, they look for equilibria in Markov strategies and establish that more information hurts both revenue and efficiency. Similar to this study, Bergemann and Hörner (2017) find that the only disclosure rule that ensures that an inefficient equilibrium does not exist is the one when only the winner's identity is disclosed.

Finally, there exists a related literature that compares various disclosure rules in a single-item auction when bidders interact post auction. ${ }^{10}$ I mention only few examples. Information disclosure about bids matters in Lebrun (2010) because bidders can engage in resale after the auction; in Giovannoni and Makris (2014) because bidders have reputational concerns; and in Fan et al. (2016) because bidders engage in oligopolistic competition after a cost-reducing patent has been auctioned to one of them.

The rest of the article is organised as follows. Section 2 sets out the model. Sections 3 and 4 analyse the cases with negative and positive correlations between the valuations of the items, respectively. Section 5 contains discussion of various extensions of the model. Finally, most of the proofs are relegated to the appendix.

\section{The model}

Two bidders are competing for two items in sequential first-price auctions. I assume that each bidder attaches to each item either a low value or a high value. The valuations of one bidder are independent of the valuations of the other bidder. However, bidder's valuations can be correlated across items. The distribution of valuations, which is the same for both bidders, is summarized in the following table:

\begin{tabular}{cc|c|c|} 
& \multicolumn{2}{c}{ Item 2} \\
\cline { 3 - 4 } Item 1 & 0 & \multicolumn{1}{c}{0} \\
\cline { 3 - 4 } & & $p_{00}$ & $p_{01}$ \\
\cline { 3 - 4 } & 1 & $p_{10}$ & $p_{11}$ \\
\cline { 3 - 4 } & & &
\end{tabular}

\footnotetext{
10 Another interesting strand of literature (for example, Esponda 2008; Jehiel 2011) considers information feedback from past auctions when bidders do not know the true distribution of types of other bidders.
} 
Without loss of generality, the low value is normalized to 0 for both items, and the high value for the first item is normalized to 1 . The high value for the second item is $v>0$. For some of the results, I will need to assume that additionally $v \leq 1$. Each bidder only knows the realization of his own valuations. I assume that bidders' payoffs are additive in the two items and money, as well as linear in money.

The items are sold sequentially. The bidders submit sealed bids for the first item and the outcome of this auction is determined. At this point, a seller might disclose some information about the outcome of the first auction. Next, the procedure is repeated for the second item. The bidder who submits the highest bid for an item, obtains it and pays his bid for that item. If there is a tie in either auction, I assume that it is broken randomly, except in the second auction when bidders have different valuations. In that case, I assume that the priority in the second auction is given to the winner of the first auction when the valuations are positively correlated, whereas the priority is given to the loser of the first auction when the valuations are negatively correlated. Intuitively, in both cases, ties are broken in favor of the bidder who is more likely to have the high value for the second item. This tie-breaking rule is needed to ensure the existence of equilibrium. ${ }^{11}$ Note, though, that the seller can implement this tie-breaking rule based on the information she observes (assuming that she knows the distribution of valuations). Finally, I do not require the bids to be non-negative, although all equilibrium strategies would remain the same if negative bids were ruled out. Since the assumption that the low value is equal to 0 is just a normalization, it is natural to allow the bidders to bid below the low value.

Before the start of the second auction, the seller has a choice how much information to release about the outcome of the first auction. The seller can choose to disclose both the winning and losing bids, only the winning bid, only the losing bid, only the winner's identity, or she can choose not to disclose anything. ${ }^{12}$ Note, though, it is assumed that the seller commits to the chosen disclosure rule before the first item is auctioned and the bidders know which rule the seller has chosen. The objective is to derive equilibrium strategies and to compare the seller's revenue from the sequential auctions under different disclosure rules.

I adopt the perfect Bayesian equilibrium as the solution concept, but additionally I also require that no bidder uses dominated strategies in the equilibrium. ${ }^{13}$ The outof-equilibrium beliefs will be specified as follows: if the equilibrium bid of a bidder in the first auction must belong to an interval $[\underline{b}, \bar{b}]$, but instead it is below $\underline{b}$ (resp., above $\bar{b}$ ), then the beliefs about this bidder will be exactly the same as the ones if he had bid $\underline{b}$ (resp., $\bar{b})$.

\footnotetext{
11 The adopted tie-breaking rule is consistent with what is done elsewhere in the literature, even though some papers make different assumptions. For example, Kannan (2012) assumes that all ties are broken randomly. But, he also additionally assumes that if bidders with different valuations tie, then the high valuation bidder, in fact, bids infinitesimally more, thus effectively breaking the tie in favour of this bidder.

12 More precisely, when either or both bids are disclosed, I additionally assume that each bidder also learns whether or not he has won the first auction. This additional assumption is only needed when ties arise with a strictly positive probability in the equilibrium of the first auction.

13 As a result of information disclosure, it can become common knowledge that one of the bidders has high value for the second item. Then, there exists an equilibrium in the second auction, in which both bidders submit bids equal to the high value for the second item even if one of them has low value. I rule out such equilibria.
} 
Rather than solving for the equilibrium strategies for all possible distributions of valuations, in the continuation, I consider two extreme cases: when the valuations are perfectly negatively correlated, and when they are perfectly positively correlated. These cases correspond to $p_{00}=p_{11}=0$ and $p_{01}=p_{10}=0$, respectively. In Sect. 5, I will also consider special cases of imperfectly correlated valuations.

\section{Perfectly negatively correlated valuations}

I first consider the case when $p_{00}=p_{11}=0$. To simplify notation, let us denote $p_{01}$ by $p$, and $p_{10}$ by $1-p$. I also assume that $p \in(0,1)$.

I start by stating the equilibrium strategies in the second auction. Let $w$ and $l$ denote the winner and the loser of the first auction, respectively. For $i=w, l$, let $q_{i}$ be the probability that bidder $i$ is of type $(1,0)$. As mentioned in the Introduction, when the valuations are negatively correlated, the winner of the first auction is perceived to be type $(1,0)$ bidder with higher probability, that is, he is the weak bidder. Therefore, I assume that $q_{w} \geq q_{l}$. Later I will verify that this assumption is indeed satisfied.

The following lemma describes the equilibrium strategies of the second auction whenever the probabilities $q_{i}$ for $i=w, l$ are common knowledge between the opponents and $q_{w} \geq q_{l}$.

Lemma 1 Type $(1,0)$ bids 0 . Type $(0,1)$ bids $v$ if $q_{w}=0$, and bids 0 if $q_{w}=1$. If $0<q_{w}<1$, then

1. Bidder $w$ of type $(0,1)$ draws a bid c according to the distribution function

$$
G_{w}(c)=\frac{q_{w}}{1-q_{w}} \frac{c}{v-c}
$$

on the interval $\left[0,\left(1-q_{w}\right) v\right]$,

2. Bidder l of type $(0,1)$ draws a bid c according to the distribution function

$$
G_{l}(c)=\frac{q_{w}}{1-q_{l}} \frac{v}{v-c}-\frac{q_{l}}{1-q_{l}}
$$

on the interval $\left[0,\left(1-q_{w}\right) v\right]$, and puts a mass $G_{l}(0)>0$ on bid 0 if $q_{w}>q_{l}$.

The equilibrium payoffs of types $(1,0)$ and $(0,1)$ are, respectively, 0 and $q_{w} v$ in the second auction.

Because versions of this lemma have already been derived in the literature (see, for example, Maskin and Riley (1985)), its proof is omitted. ${ }^{14}$

Lemma 1 says that a type $(0,1)$ bidder, irrespective of whether or not he has won the first auction, expects a payoff of $q_{w} v$ in the second auction. The results of Sect. 3 that follow can be interpreted in terms of this payoff. First, if the wining bid of the first auction is disclosed, the loser will use this bid to update his belief, $q_{w}$, about the opponent. Otherwise, he will use his own losing bid to update his belief. This explains

$\overline{14}$ This lemma is also a special case of Lemma 15 in Āzacis (2019) with $n \geq 2$ bidders. 
why certain disclosure rules are equivalent. Second, as I will show, $q_{w}$ is increasing in the bids of the first auction whenever the seller discloses something about the outcome of the first auction. This gives the incentives to a type $(0,1)$ bidder to bid more aggressively in the first auction, which benefits the seller. Third, if $0<q_{w}<1$ holds when the winning bid is disclosed, the equilibrium outcome of the first auction must be (partially) pooling and, hence, inefficient.

Let $F_{01}(b)$ and $F_{10}(b)$ denote the equilibrium strategies of types $(0,1)$ and $(1,0)$, respectively, in the first auction. Also, let $b_{w}$ and $b_{l}$ denote the winning and losing bids of the first auction, respectively.

\subsection{When no information is disclosed}

I start by characterizing the equilibrium strategies when no information is disclosed. This case will serve as a benchmark. Although the assumption that a bidder does not even learn whether or not he has won the first auction, appears extreme, note that we can think that both items are sold simultaneously.

\section{Proposition 2}

Item 1: Type $(0,1)$ bids 0 and type $(1,0)$ draws a bid according to $F_{10}$ : $[0,1-p] \rightarrow[0,1]$, where

$$
F_{10}(b)=\frac{p}{1-p} \frac{b}{1-b} .
$$

Item 2: The bidders bid as specified in Lemma 1 , where $q_{w}=q_{l}=1-p .^{15}$

The equilibrium payoffs of types $(1,0)$ and $(0,1)$ are, respectively, $\pi_{10}=p$ and $\pi_{01}=(1-p) v$.

Because no information is disclosed between the two auctions, they can be treated as two independent auctions. As a result, the equilibrium bidding in the first auction is similar to the one described in Lemma 1 (with the strategies of types $(1,0)$ and $(0,1)$ exchanged, and setting $v=1$ and $q_{w}=q_{l}=p$ ).

The equilibrium of the sequential auctions is efficient with the surplus equal to $(1-p)^{2}+p^{2} v+2 p(1-p)(1+v)$, the ex ante payoff of a bidder is $p(1-p)(1+v)$, and the equilibrium revenue of the seller, which is the difference between the surplus and the bidders' payoffs, is equal to $(1-p)^{2}+p^{2} v$.

\subsection{Only the winning bid is disclosed}

Before stating the equilibrium strategies of the first auction formally, I describe them in words. The equilibrium strategies are also illustrated for specific parameter values in Fig. 1. Type $(0,1)$ bidder bids 0 with a positive probability, which is less than 1.

15 Because $q_{w}=q_{l}$, it does not matter that the bidder does not know whether he is the winner or the loser of the first auction. 

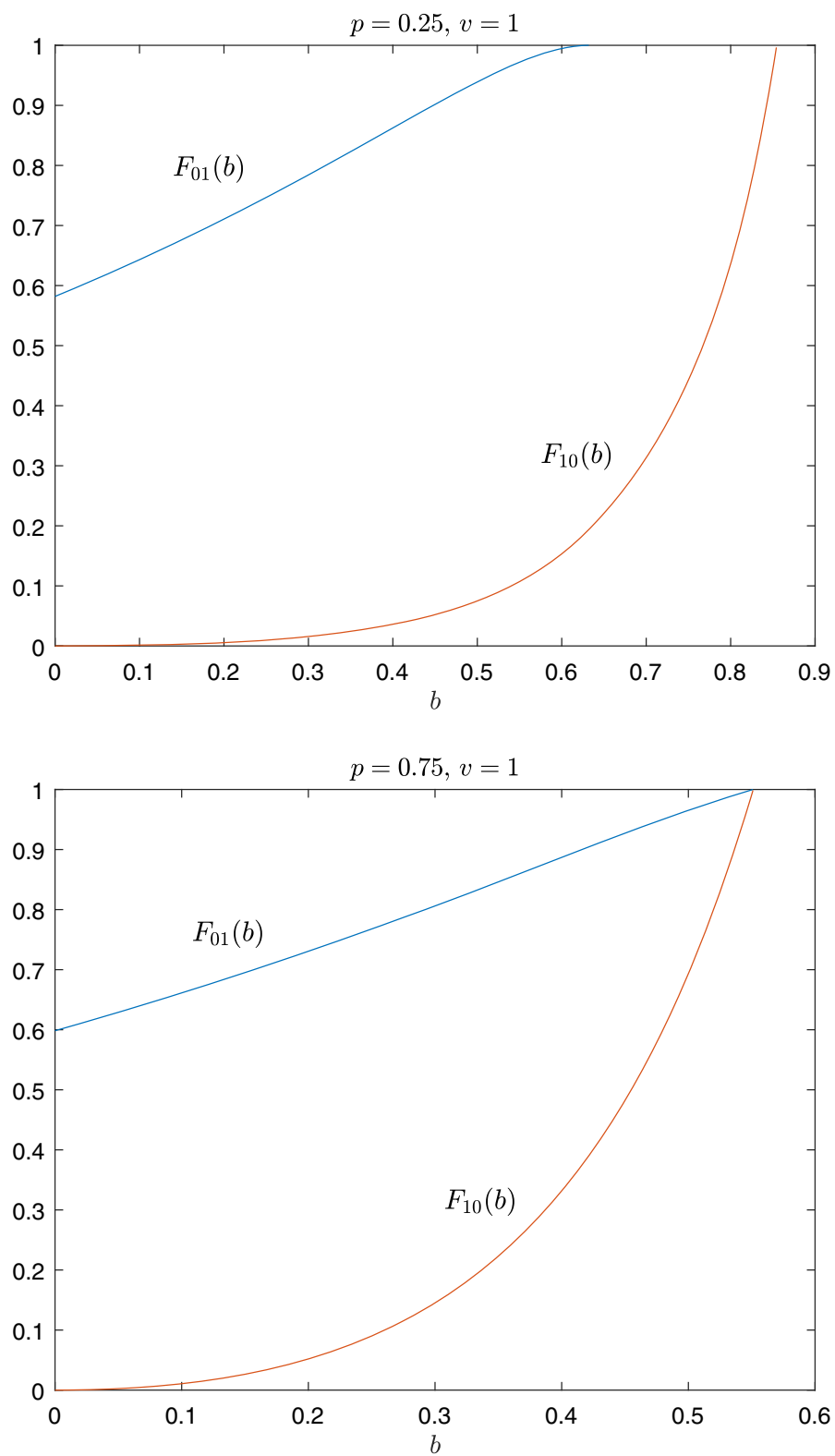

Fig. 1 Equilibrium strategies

With the remaining probability, he bids above 0 , i.e., above his valuation of the first item. Type $(1,0)$ bids above 0 with probability 1 . Depending on the parameter values, either both types randomize on the same interval or the support of type $(0,1)$ bids is a strict subset of the support of type $(1,0)$ bids. Because type $(0,1)$ can win the first auction against type $(1,0)$ with a strictly positive probability, the equilibrium is inefficient. 


\section{Proposition 3}

Item 1: $F_{01}:[0, \bar{b}] \rightarrow[0,1]$, where

$$
F_{01}(b)=F_{01}(0) \frac{v+b+\ln (1-b)}{v(1-b)} .
$$

If $p v \geq 1-e^{-v}$, then $F_{10}:[0, \bar{b}] \rightarrow[0,1]$, where

$$
F_{10}(b)=-F_{01}(0) \frac{p}{1-p} \frac{b+\ln (1-b)}{v(1-b)},
$$

$F_{01}(0)=\frac{1-\bar{b}}{p}$ and $\bar{b}$ is given by $v(1-p)+\bar{b}+\ln (1-\bar{b})=0$.

If $p v<1-e^{-v}$, then $F_{10}:\left[0,1-\frac{p v}{e^{v}-1}\right] \rightarrow[0,1]$, where $F_{10}(b)$ is defined in (3) for $b \in[0, \bar{b}]$, and it is

$$
F_{10}(b)=F_{01}(0) \frac{p}{1-p} \frac{1}{1-b}-\frac{p}{1-p}
$$

for $b \in\left(\bar{b}, 1-\frac{p v}{e^{v}-1}\right], F_{01}(0)=\frac{v}{e^{v}-1}<1$ and $\bar{b}=1-e^{-v}$.

Item 2: The bidders bid as specified in Lemma 1, where for $0 \leq b_{w} \leq \bar{b},{ }^{16}$

$$
\begin{aligned}
q_{w} & =\frac{(1-p) f_{10}\left(b_{w}\right)}{p f_{01}\left(b_{w}\right)+(1-p) f_{10}\left(b_{w}\right)}=-\frac{\ln \left(1-b_{w}\right)}{v}, \\
q_{l} & =\frac{(1-p) F_{10}\left(b_{w}\right)}{p F_{01}\left(b_{w}\right)+(1-p) F_{10}\left(b_{w}\right)}=-\frac{b_{w}+\ln \left(1-b_{w}\right)}{v} .
\end{aligned}
$$

The equilibrium payoffs of types $(1,0)$ and $(0,1)$ are, respectively, $\pi_{10}=p F_{01}(0)$ and $\pi_{01}=(1-p) v$.

The proof of this and other propositions that are omitted from the main text, can be found in Appendix A.

The ex ante payoff of a bidder is

$$
p \pi_{01}+(1-p) \pi_{10}=p(1-p)\left(v+F_{01}(0)\right) .
$$

Thus, the payoff is lower compared with the situation when no information is released. The surplus from the auction is

$$
\begin{aligned}
& (1-p)^{2} \times 1+p^{2} \times 0+2 p(1-p) \times \int_{0}^{\bar{b}_{10}} F_{01}(b) f_{10}(b) d b \\
& \quad+(1-p)^{2} \times 0+p^{2} \times v+2 p(1-p) \times v,
\end{aligned}
$$

16 When $b_{w}=0, q_{w}=\frac{(1-p) f_{10}(0)}{p F_{01}(0)+(1-p) f_{10}(0)}=0$. If $b_{w}>\bar{b}$, then set $q_{w}=\frac{(1-p) f_{10}(\bar{b})}{p f_{01}(\bar{b})+(1-p) f_{10}(\bar{b})}$. 
where the first line is the surplus from the first auction and the second line is the surplus from the second auction, $\bar{b}_{10}=\bar{b}$ if $p v \geq 1-e^{-v}$ and $\bar{b}_{10}=1-\frac{p v}{e^{v}-1}$ otherwise. Clearly, because the equilibrium is inefficient, the surplus of the first auction is not maximized. The revenue of the seller is the difference between the surplus and the bidders' payoffs, and is given by

$$
R_{n}^{w}=(1-p)^{2}+p^{2} v+2 p(1-p)\left(\int_{0}^{\bar{b}_{10}} F_{01}(b) f_{10}(b) d b-F_{01}(0)\right)
$$

Because $F_{01}(b) \geq F_{01}(0)$ for all $b$, the revenue exceeds the one under no disclosure.

\subsection{The winning and losing bids are disclosed}

It turns out that in this case, the equilibrium payoffs of the bidders and the equilibrium revenue of the seller are the same as in the case when only the winning bid is disclosed. Even more, the equilibrium strategies of both types in the first auction are exactly the same for both disclosure rules. The only change in the strategy is for type $(0,1)$ bidder in the second auction after he has lost the first auction. The intuition for this result is simple. The expected payoff of type $(0,1)$ bidder in the second auction only depends on the value of $q_{w}$. Whether or not the losing bid of the first auction is also disclosed does not affect this value. Therefore, the first auction's strategies are unaffected by the decision to disclose the losing bid in addition to the winning bid.

Proposition 4 The equilibrium strategies are the same as in Proposition 3, except that for $0 \leq b_{l} \leq \bar{b},{ }^{17}$

$$
q_{l}=\frac{(1-p) f_{10}\left(b_{l}\right)}{p f_{01}\left(b_{l}\right)+(1-p) f_{10}\left(b_{l}\right)}=-\frac{\ln \left(1-b_{l}\right)}{v} .
$$

Proof One only needs to verify two things. First, the winner's belief about the opponent's type at the start of the second auction, as described by $q_{l}$, is indeed derived from the strategies of the first auction using Bayes' formula. Second, the requirement that $q_{l} \leq q_{w}$ is also satisfied.

\subsection{Only the losing bid is disclosed}

The equilibrium bidding strategies in this and next subsection are only valid for $v \leq$ $1 .^{18}$ The following proposition establishes that in the first auction, types $(0,1)$ and $(1,0)$ randomize on adjacent intervals. Therefore, the type of the loser of the first auction (and sometimes the type of the winner) is fully revealed prior to the second

17 If $b_{l}>\bar{b}$, then $q_{l}=\frac{(1-p) f_{10}(\bar{b})}{p f_{01}(\bar{b})+(1-p) f_{10}(\bar{b})}$.

18 For $v>1$, I failed to identify equilibrium strategies in the first auction. Even if one can restore the existence of equilibrium by assuming a different tie-breaking rule or by allowing an equilibrium in weakly dominated strategies (see Footnote 13), such an equilibrium would not be comparable with the one when the winning bid is announced. Therefore, I only present the equilibrium strategies for $v \leq 1$. 
auction. Also, the equilibrium is efficient, even though type $(0,1)$ bids strictly above his valuation for the first item.

Proposition 5 Suppose $v \leq 1 .^{19}$

Item 1: $F_{01}:\left[(1-p) v,-\ln (1-p) \frac{1-p}{p} v\right] \rightarrow[0,1]$, where $F_{01}$ is implicitly defined by

$$
\begin{gathered}
p F_{01}(b)(-b)=(1-p) \ln \left(1-p F_{01}(b)\right) v \\
F_{10}:\left[-\ln (1-p) \frac{1-p}{p} v,(1-p)(1-\ln (1-p) v)\right] \rightarrow[0,1], \text { where } \\
F_{10}(b)=\frac{p}{1-p} \frac{b+\ln (1-p) \frac{1-p}{p} v}{1-b} .
\end{gathered}
$$

Item 2: The bidders bid as specified in Lemma 1, where $q_{l}=0$ if $b_{l} \leq$ $-\ln (1-p) \frac{1-p}{p} v$ and $q_{l}=1$ if $b_{l}>-\ln (1-p) \frac{1-p}{p} v$, whereas

$$
q_{w}=\frac{1-p}{1-p+p\left(1-F_{01}\left(b_{l}\right)\right)} .
$$

The equilibrium payoffs of types $(1,0)$ and $(0,1)$ are, respectively, $\pi_{10}=p+$ $(1-p) \ln (1-p) v$ and $\pi_{01}=(1-p) v$.

Given the equilibrium strategies, the ex ante payoff of a bidder is

$$
p \pi_{01}+(1-p) \pi_{10}=p(1-p)(1+v)+(1-p)^{2} \ln (1-p) v .
$$

Because the equilibrium of the auction is efficient, the surplus from the auction is $(1-p)^{2}+p^{2} v+2 p(1-p)(1+v)$. The revenue of the seller is

$$
R_{n}^{l}=(1-p)^{2}+p^{2} v-2(1-p)^{2} \ln (1-p) v .
$$

\subsection{Only the winner's identity is disclosed}

Now, when deriving the equilibrium strategies, one cannot apply the results of Lemma 1 to the second auction because the values of $q_{w}$ and $q_{l}$ will not be common knowledge between the bidders. For example, the belief of the loser of the first item that the opponent has low valuation for the second item will depend on the loser's bid, which is known to him but unknown to the opponent. In order to define the equilibrium

19 Given this restriction, one can verify that $-\ln (1-p) \frac{1-p}{p} v<(1-p)(1-\ln (1-p) v)$ or, equivalently, $p+(1-p) \ln (1-p) v>0$ holds. Likewise, $(1-p) v<-\ln (1-p) \frac{1-p}{p} v$ or, equivalently, $p+\ln (1-p)<0$ holds. Therefore, the supports of the equilibrium strategies are well-defined. 
strategies of the second auction in the proposition below, I introduce the following function:

$$
c(b)=\frac{p\left(1-F_{01}(b)\right)}{1-p+p\left(1-F_{01}(b)\right)} v,
$$

where $F_{01}(b)$ is defined in (5) and $b$ takes values in the interval $\left[(1-p) v,-\ln (1-p) \frac{1-p}{p} v\right]$. This function will define a mapping from the bids $b$ of the first auction into the bids $c$ of the second auction. To understand its properties, let $H_{w}(c) \equiv \frac{1-p}{p} \frac{c}{v-c}$ for $c \in[0, p v]$. Then, one can verify that $1-F_{01}(b)=H_{w}(c(b))$ holds. Further, because $H_{w}^{\prime}(c)>0$ and $F_{01}^{\prime}(b)>0$, it follows that $c^{\prime}(b)<0$. Thus, $c(b)$ is monotonically decreasing in $b$, and takes values in the interval $[0, p v] .{ }^{20}$

Proposition 6 Suppose $v \leq 1$.

Item 1: The bidders bid as in Proposition 5.

Item 2: Type $(1,0)$ bids 0 . Bidder $w$ of type $(0,1)$ bids $c_{w}$ which is defined by (7) for $b=b_{w}$. Bidderl of type $(0,1)$ draws a bid c from the interval $\left[0,\left(1-q_{w}\right) v\right]$ according to

$$
H_{l}(c)=\frac{q_{w} v}{v-c}
$$

where $q_{w}$ is defined in (6). ${ }^{21}$ The equilibrium payoffs of types $(1,0)$ and $(0,1)$ are, respectively, 0 and $q_{w} v$ in the second auction.

Thus, in the equilibrium of the second auction, type $(0,1)$ loser of the first auction draws a random bid from an interval (as is usual in the first-price auction with binary valuations), but type $(0,1)$ winner of the first auction submits a bid, which is a monotone transformation of his bid in the first auction. Though, as shown in the proof, from the perspective of type $(0,1)$ loser, it is as if type $(0,1)$ winner is randomizing on the interval $\left[0,\left(1-q_{w}\right) v\right]$ according to $\frac{H_{w}(c)}{H_{w}\left(\left(1-q_{w}\right) v\right)}$ in the second auction. As a result, type $(0,1)$ bidder, either he wins or loses the first auction, expects the same payoff of $q_{w} v$ in the second auction (which he could guarantee by submitting a bid of $\left(1-q_{w}\right) v$ ). Interestingly, the winner of the first auction does not know the value of $q_{w}$. However, his strategy in the second auction does not depend on this value. Furthermore, because the expression for $q_{w}$ is the same as the one when only the losing bid is disclosed, the equilibrium strategies of the first auction are also the same under both disclosure rules. As a result, the equilibrium outcome of the sequential auctions when only the

20 The inverse of function $c(b)$ is

$$
b(c)=-\ln \left(\frac{(1-p) v}{1-c}\right) \frac{(1-p)(v-c)}{p v-c} v .
$$

21 If type $(0,1)$ submitted a bid $b \notin\left[(1-p) v,-\ln (1-p) \frac{1-p}{p} v\right]$ in the first auction, then he bids in the second auction as if he had submitted $b=(1-p) v$ or $b=-\ln (1-p) \frac{1-p}{p} v$, whichever is closer to his actual bid in the first auction. 
winner's identity is disclosed, is also equivalent to the one when only the losing bid is disclosed. ${ }^{22}$

\subsection{The ranking of revenues}

Given the outcome equivalence between various disclosure rules, it is enough to compare the cases when only the winner's bid or only the loser's bid is disclosed (when $v \leq 1$ ). The revenue from the auction when the winner's bid is disclosed is higher than the revenue when only the loser's bid is disclosed, $R_{n}^{w} \geq R_{n}^{l}$ if $^{23}$

$$
\Delta_{n}=p\left(\int_{0}^{\bar{b}_{10}} F_{01}(b) f_{10}(b) d b-F_{01}(0)\right)+(1-p) \ln (1-p) v \geq 0 .
$$

$\Delta_{n}=0$ is plotted in Fig. 2. For the $(p, v)$ pairs to the right of $\Delta_{n}=0$ curve, it is optimal for the seller to disclose the winning bid, and for the $(p, v)$ pairs to the left of $\Delta_{n}=0$ curve, it is optimal to disclose the losing bid. Further, if $p \leq p^{*} \approx 0.7153$, then it is not optimal to disclose the winning bid for any value of $v \leq 1$. The reason why $R_{n}^{l}$ is higher for most of the parameter values, can be partly attributed to the inefficiency of the equilibrium when the winning bid is disclosed. The main reason, however, is that for most parameter values, the bidders appear to bid more aggressively and, consequently, expect a lower payoff when the losing bid is disclosed. This is shown in Fig. 2 by $\delta_{n}=0$, where $\delta_{n}=-\left(p(1-p)\left(1-F_{01}(0)\right)+(1-p)^{2} \ln (1-p) v\right)$ measures the difference in ex ante payoffs of a bidder when the winning bid and the losing bid are, respectively, disclosed. For the $(p, v)$ pairs to the left of $\delta_{n}=0$ curve, bidder's payoff is higher when the seller discloses the winning bid.

\section{Perfectly positively correlated valuations}

I now consider the case when $p_{01}=p_{10}=0$. As noted in the Introduction, this case has already been studied in the literature for some disclosure rules although assuming $v=1$. To simplify notation, let us now denote $p_{00}$ by $p$, and $p_{11}$ by $1-p$. I again assume that $p \in(0,1)$. Below I am going to establish a similar set of results as in the case of negatively correlated values.

As before, let $w$ and $l$ be the winner and the loser of the first auction, respectively. For $i=w, l$, let $q_{i}$ now be the probability that bidder $i$ is of type $(0,0)$. Next, I restate Lemma 1 but now I assume that $q_{w} \leq q_{l}$. That is, I assume that the loser of the first auction is now the weak bidder. I will verify that $q_{w} \leq q_{l}$ indeed holds once the strategies for the first auction are stated.

\footnotetext{
22 One can also note that $\frac{H_{w}(c)}{H_{w}\left(\left(1-q_{w}\right) v\right)}=G_{w}(c)$, which is given in (1), and $H_{l}(c)=G_{l}(c)$, which is given in (2) when $q_{l}=0$. Thus, the equilibrium distribution of bids in the second auction is the same as the one implied by Lemma 1.

$23 F_{01}, f_{10}, \bar{b}_{10}$, and $\bar{b}$ refer to the functions and variables that are defined in Sect. 3.2. $\Delta_{n}$ is explicitly calculate in Appendix B.
} 


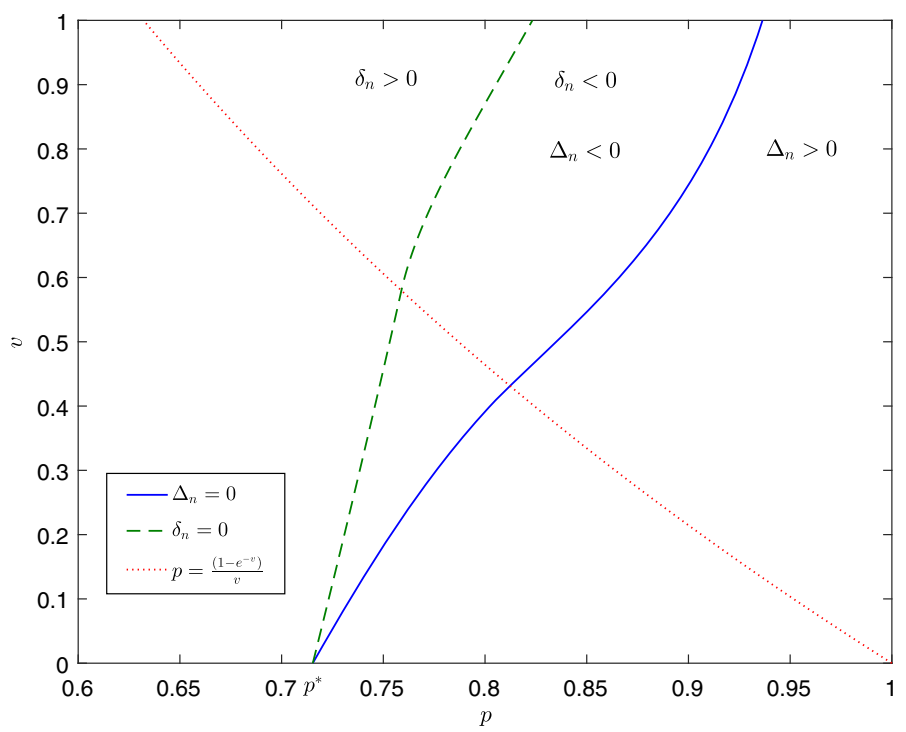

Fig. 2 The ranking of revenues

Lemma 7 Type $(0,0)$ bids 0 . Type $(1,1)$ bids $v$ if $q_{l}=0$, and bids 0 if $q_{l}=1$. If $0<q_{l}<1$, then

1. Bidder $w$ of type $(1,1)$ draws a bid c according to the distribution function

$$
G_{w}(c)=\frac{q_{l}}{1-q_{w}} \frac{v}{v-c}-\frac{q_{w}}{1-q_{w}}
$$

on the interval $\left[0,\left(1-q_{l}\right) v\right]$, and puts a mass $G_{w}(0)>0$ on bid 0 if $q_{w}<q_{l}$.

2. Bidder l of type $(1,1)$ draws a bid c according to the distribution function

$$
G_{l}(c)=\frac{q_{l}}{1-q_{l}} \frac{c}{v-c}
$$

on the interval $\left[0,\left(1-q_{l}\right) v\right]$.

The equilibrium payoffs of types $(0,0)$ and $(1,1)$ are, respectively, 0 and $q_{l} v$ in the second auction.

Similar to the case with negatively correlated valuations, the results that follow can be interpreted in terms of the payoff that a type $(1,1)$ bidder expects in the second auction. This payoff now depends on $q_{l}$. Therefore, the equivalence between different disclosure rules changes. Now, whenever the losing bid of the first auction is disclosed, the winner will use this bid to calculate $q_{l}$. Otherwise, he will use his own winning bid. Also, as will be shown, $q_{l}$ is decreasing in the bids of the first auction whenever the seller discloses something about the outcome of the first auction. Therefore, a type $(1,1)$ bidder has incentives to bid less aggressively in the first auction, which hurts the 
seller's revenue. Finally, whenever the losing bid is disclosed and $0<q_{l}<1$ holds, the equilibrium outcome of the first auction must be pooling and, hence, inefficient.

For all the disclosure rules that I am going to consider, it is true that type $(0,0)$ bids 0 in the equilibrium of the first auction. ${ }^{24}$ That it is indeed an equilibrium strategy will follow from the fact that the lowest bid that type $(1,1)$ submits in equilibrium, is more or equal to 0 . Therefore, I will omit the strategy of type $(0,0)$ bidder from the propositions that follow. I also denote the equilibrium strategy of type $(1,1)$ in the first auction by $F(b)$.

\subsection{When no information is disclosed}

The equilibrium strategies are exactly the same as in Proposition 2 with types $(0,1)$ and $(1,0)$ replaced by types $(0,0)$ and $(1,1)$, respectively, and $q_{l}=q_{w}=p$. The equilibrium is efficient, the surplus is $\left(1-p^{2}\right)(1+v)$, the ex ante payoff of a bidder is $p(1-p)(1+v)$, and the equilibrium revenue of the seller is equal to $(1-p)^{2}(1+v)$.

\subsection{Only the winning bid is disclosed}

Here I assume that $v \leq 1 .^{25}$

Proposition 8 Suppose $v \leq 1$.

Item 1: $F:[0,1-p+v p \ln p] \rightarrow[0,1]$, where $F$ is implicitly defined by ${ }^{26}$

$$
(p+(1-p) F(b))(1-b)-v p \ln (p+(1-p) F(b))=p(1-v \ln p) .
$$

Item 2: The bidders bid as specified in Lemma 7, where $q_{l}=q_{w}=1$ if $b_{w}=0$, otherwise $q_{w}=0$, and ${ }^{27}$

$$
q_{l}=\frac{p}{p+(1-p) F\left(b_{w}\right)}
$$

The equilibrium payoff of type $(1,1)$ is $p(1+v(1-\ln p))$.

One can verify that $F(0)=0$, which means that the equilibrium is efficient and the surplus is $\left(1-p^{2}\right)(1+v)$. The ex ante equilibrium payoff of a bidder is $p(1-p)(1+$ $v(1-\ln p))$. Hence, the seller's revenue is given by

$$
R_{p}^{w}=(1-p)^{2}(1+v)+2 p(1-p) v \ln p
$$

\footnotetext{
24 Note that I allow the bids to be negative.

${ }^{25}$ For $v>1$, I again failed to identify equilibrium strategies in the first auction, given the assumed tie-breaking rule and the equilibrium strategies in the second auction as specified in Lemma 7.

26 That $F$ is monotonically increasing is verified in the next subsection. There I need the assumption that $v \leq 1$.

27 If $b_{w}>1-p+v p \ln p$, then $q_{l}=p$.
} 
It follows that the bidders gain and the seller loses compared to the case when no information is revealed.

\subsection{Only the winner's identity is disclosed}

Because the values of $q_{i}$ for $i=w, l$ are not common knowledge between the bidders when only the winner's identity is disclosed, one cannot apply the results of Lemma 7 to the second auction. In order to define the equilibrium strategies of the second auction, I introduce the following function:

$$
b(c)=\frac{c}{v}+(v-c) \ln \left(1-\frac{c}{v}\right)
$$

for $c \in[0,(1-p) v]$. It will define an implicit mapping from the bids $b$ of the first auction into the bids $c$ of the second auction. First, observe that the function in (12) is monotonically increasing,

$$
\frac{d b}{d c}=\frac{1-v}{v}-\ln \left(1-\frac{c}{v}\right)>0
$$

given that $0 \leq c<v \leq 1$, and $b$ takes values in $[0,1-p+v p \ln p]$. Second, if one defines a distribution function $H_{l}(c) \equiv \frac{p}{1-p} \frac{c}{v-c}$ for $c \in[0,(1-p) v]$, then one can verify that $F(b(c))=H_{l}(c)$ holds for $F$ defined in (10). (Also, $F^{\prime}(b) b^{\prime}(c)=H_{l}^{\prime}(c)$. Because $b^{\prime}(c)>0$ and $H_{l}^{\prime}(c)>0$, then indeed $F^{\prime}(b)>0$ in (10) as required.) From $F(b)=\frac{p}{1-p} \frac{c}{v-c}$, one can rewrite (12) as

$$
c=\frac{(1-p) F(b)}{p+(1-p) F(b)} v
$$

Proposition 9 Suppose $v \leq 1$.

Item 1: The bidders bid as in Proposition 8.

Item 2: Type $(0,0)$ bids 0 . Bidder l of type $(1,1)$ bids $c_{l}$, which is defined by (13) for $b=b_{l}$. Bidder $w$ of type $(1,1)$ draws a bid $c$ from the interval $\left[0, c_{w}\right]$ according to

$$
H_{w}(c)=\frac{v-c_{w}}{v-c}
$$

where $c_{w}$ is defined by (13) for $b=b_{w} \cdot{ }^{28}$ The equilibrium payoffs of types $(0,0)$ and $(1,1)$ are, respectively, 0 and $v-c_{w}=q_{l} v$ in the second auction where $q_{l}$ is given by (11).

28 If type $(1,1)$ submitted a bid $b \notin[0,1-p+v p \ln p]$ in the first auction, then he bids in the second auction as if he had submitted $b=0$ or $b=1-p+v p \ln p$, whichever is closer to his actual bid in the first auction. 
Note that the winner of the first auction does not know what $c_{l}$ is, but he knows that it is less than $c_{w}$ and that the distribution of $c_{l}$ is $\frac{H_{l}\left(c_{l}\right)}{H_{l}\left(c_{w}\right)}$, provided that the opponent's type is $(1,1)$. Therefore, from the perspective of the winner, type $(1,1)$ loser effectively randomizes as specified in Lemma 7 . Because type $(1,1)$ winner also randomizes as specified in Lemma 7 (where $q_{w}=0$ ), the strategies in the second auction form an equilibrium, in which both expect a payoff of $v-c_{w}=q_{l} v$. Further, conditional on the bids of the first auction, the value of $q_{l}$ is the same whether the winning bid or the winner's identity are disclosed. This implies that the equilibrium strategies in the first auction are also the same for both disclosure rules. Thus, one can conclude that disclosing only the winning bid and disclosing only the winner's identity are outcome equivalent. Note, though, that the equivalence result was different when the valuations were negatively correlated. In that case, disclosing the winner's identity was equivalent to disclosing only the losing bid.

\subsection{Only the losing bid is disclosed}

Here and in the next subsection, I do not require that $v \leq 1$.

\section{Proposition 10}

Item 1: If $p<\frac{2 v}{1+2 v}$, then $F:[0,1+p v-\sqrt{p v(2+p v)}] \rightarrow[0,1]$, where

$$
\begin{gathered}
F(b)=\frac{p}{1-p} \frac{b}{1-b}+\frac{\sqrt{p v(2+p v)}-p(1+v)}{1-p} \frac{1}{1-b} . \\
\text { If } \frac{2 v}{1+2 v} \leq p<1, \text { then } F:[0,1-p] \rightarrow[0,1], \text { where } \\
F(b)=\frac{p}{1-p} \frac{b}{1-b} .
\end{gathered}
$$

Item 2: The bidders bid as specified in Lemma 7, where $q_{w}=q_{l}=0$ if $b_{l}>0$; otherwise

$$
\begin{aligned}
q_{w} & =\frac{\frac{1}{2} p}{\frac{1}{2}(p+(1-p) F(0))+(1-p)(1-F(0))}, \\
q_{l} & =\frac{p}{p+(1-p) F(0)} .
\end{aligned}
$$

The equilibrium payoff of type $(1,1)$ is $\sqrt{p v(2+p v)}$ when $p<\frac{2 v}{1+2 v}$ and it is $p(1+v)$ when $\frac{2 v}{1+2 v} \leq p<1$.

Note that $F(0)=0$ in (14) when $p=\frac{2 v}{1+2 v}$. Therefore, the first expression for $F$ only applies when $p<\frac{2 v}{1+2 v}$. In this case, type $(1,1)$ bids 0 with a strictly positive probability and, as a result, can lose to type $(0,0)$ opponent. Therefore, the equilibrium is inefficient when $p<\frac{2 v}{1+2 v}$. 
When $\frac{2 v}{1+2 v} \leq p<1$, the bidders' payoffs and the seller's revenue in the equilibrium are the same as in the case of no information disclosure. That is, the ex ante payoff of a bidder is given by $p(1-p)(1+v)$ and the seller's revenue is given by

$$
R_{p}^{l}=(1-p)^{2}(1+v)
$$

When $p<\frac{2 v}{1+2 v}$, the ex ante equilibrium payoff of a bidder is $(1-p) \sqrt{p v(2+p v)}$. The surplus in the first auction will be 0 if a bidder with 0 valuation wins that auction. This happens with a probability of

$$
p^{2}+2 p(1-p) F(0) \times \frac{1}{2}=p(\sqrt{p v(2+p v)}-p v) .
$$

The surplus in the second auction is 0 if both bidders have 0 valuations, which happens with a probability of $p^{2}$. Hence, the surplus from both auctions is

$$
1-p(\sqrt{p v(2+p v)}-p v)+\left(1-p^{2}\right) v=1+v-p \sqrt{p v(2+p v)} .
$$

Finally, the seller's revenue is given by

$$
\begin{aligned}
R_{p}^{l} & =1+v-p \sqrt{p v(2+p v)}-2(1-p) \sqrt{p v(2+p v)} \\
& =1+v-(2-p) \sqrt{p v(2+p v)} .
\end{aligned}
$$

\subsection{The winning and losing bids are disclosed}

It turns out that the equilibrium outcome is equivalent to the one found in the case when only the losing bid is disclosed, again, because the formula for $q_{l}$ remains unchanged.

Proposition 11 The equilibrium strategies are the same as in Proposition 10, except that

$$
q_{i}= \begin{cases}\frac{p}{p+(1-p) F(0)} & \text { if } b_{w}=0 \\ 0 & \text { if } b_{l}>0\end{cases}
$$

for $i=w, l$, and $q_{w}=0$ and $q_{l}=\frac{p}{p+(1-p) F(0)}$ if $0=b_{l}<b_{w}$.

Proof It is easy to verify that the beliefs $q_{i}$ for $i=w, l$ are consistent with the strategies in the first auction, and that $q_{w} \leq q_{l}$ indeed holds. The only difference from Proposition 10 is in the value of $q_{w}$, but it does not affect the expected payoffs in the second auction. Therefore, the equilibrium strategies in the first auction are exactly the same as in Proposition 10.

\subsection{The ranking of revenues}

Again, given the outcome equivalence between several disclosure rules, it is enough to consider the seller's revenue for the following cases: no information is disclosed, 
only the winning bid is disclosed, and only the losing bid is disclosed. First of all, one can verify that the seller's revenue under non-disclosure, $(1-p)^{2}(1+v)$, (weakly) exceeds both $R_{p}^{w}$ and $R_{p}^{l}$. Thus, if the valuations are perfectly positively correlated, the best that the seller can do, is not to disclose any information between the first and second auctions. Alternatively, she can sell both objects in a simultaneous auction, in which the bidders either submit separate bids for each object, or they each submit a single bid for the bundle of both objects.

One may also want to compare $R_{p}^{w}$ and $R_{p}^{l}$. Here I assume that $v \leq 1$. Let $\Delta_{p}=$ $R_{p}^{w}-R_{p}^{l}$. Then, $\Delta_{p}$ is given by

$\Delta_{p}=\left\{\begin{array}{lr}(2-p)(\sqrt{p v(2+p v)}-p(1+v))+2 p(1-p) v \ln p & \text { if } \quad p<\frac{2 v}{1+2 v} \\ 2 p(1-p) v \ln p & \text { if } \frac{2 v}{1+2 v} \leq p<1 .\end{array}\right.$

It immediately follows that $\Delta_{p}<0$ when $\frac{2 v}{1+2 v} \leq p<1 . \Delta_{p}=0$ is plotted in Fig. 3 . For the values of $p$ and $v$ to the left of $\Delta_{p}=0$, disclosing the winning bid is better, otherwise disclosing the losing bid is better. In particular, if $p$ exceeds $p^{*} \approx 0.2325$, disclosing the losing bid is better for all values of $v$. The intuition for the ranking of revenues is simple when $\frac{2 v}{1+2 v} \leq p<1$ : although the surplus under both disclosure rules is the same, the bidders expect higher payoff when the winning bid is disclosed. On the other hand, if $p<\frac{2 v}{1+2 v}$, the surplus is not maximized when the losing bid is disclosed. This should work in favour of disclosing the winning bid. However, for many parameter values, this effect is reversed by the fact that the bidders also bid more aggressively and, consequently, expect lower payoffs when the losing bid is disclosed. This is illustrated in Fig. 3 by $\delta_{p}=0$ where $\delta_{p}=p(1+v(1-\ln p))-$ $\sqrt{p v(2+p v)}$ is the difference in a bidder's payoff when the winning bid and the losing bid, respectively, are disclosed.

\section{Discussion}

I have established relationships between different disclosure rules and compared them in terms of the seller's revenue and efficiency. In this section, I discuss if and how the results change if we relax the assumptions of the model. Specifically, I will consider what happens if the valuations are imperfectly correlated across items; if they are asymmetrically distributed across bidders; and if there are more then two bidders. I will mainly focus on the cases when only the winning bid is announced and when nothing is announced. I will also consider an example of more sophisticated disclosure rule. For simplicity, I set $v=1$ in this section. The results of this section are formally stated and proven in the working paper version of the article (Āzacis 2019, Appendix C).

\subsection{Imperfect correlation of valuations}

It has been found that the seller benefits from revealing some information about the outcome of the first auction prior to the second auction if the valuations are perfectly 


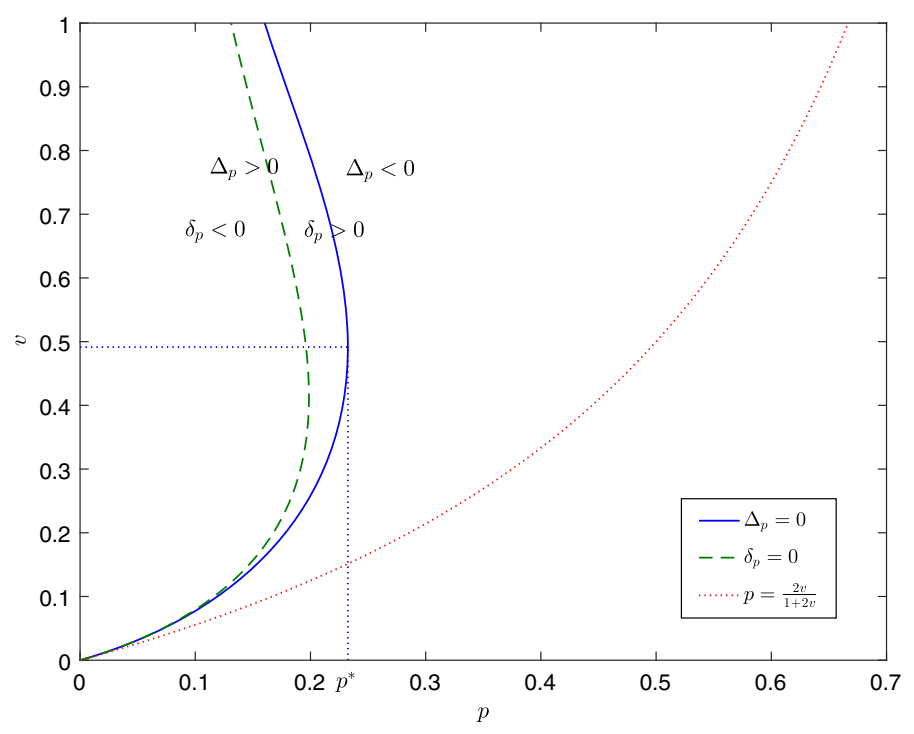

Fig. 3 The ranking of revenues

negatively correlated, but she is better off not to reveal anything if the valuations are perfectly positively correlated. Furthermore, if the valuations are not correlated across items, then the disclosure rule is irrelevant. This suggests that the results that have been established for perfect correlation of valuations, might continue to hold for imperfect correlation of valuations as long as the sign of correlation does not change. To verify this intuition, I consider two special cases.

First, I assume that the valuations of each bidder are distributed across items as follows: $p_{10}=p_{01}=\epsilon$ and $p_{00}=p_{11}=\frac{1}{2}-\epsilon$ where $0 \leq \epsilon \leq \frac{1}{4} \cdot \epsilon=0$ corresponds to the case analysed in Sect. 3, while $\epsilon=\frac{1}{4}$ implies that the valuations across items are independently distributed. When $0<\epsilon<\frac{1}{4}$, the valuations of the two items are negatively, but imperfectly correlated. In this case, each bidder can be one of four types with a strictly positive probability. If the winning bid of the first auction is revealed, I find that in the first auction, type $(0,1)$ randomizes on $[0, \underline{b}]$ interval, type $(1,1)$ randomizes on adjacent $[\underline{b}, \bar{b}]$ interval, type $(1,0)$ randomizes on $[0, \bar{b}]$ interval, while type $(0,0)$ bids 0 . (See the top panel in Fig. 4.) In the limit, when $\epsilon$ goes to 0 , the equilibrium converges to the one given in Proposition 3 for the case when $p v<1-e^{-v}$ (with $p=\frac{1}{2}$ and $v=1$ ). Since type $(0,1)$ wins with a strictly positive probability when facing type $(1,0)$ opponent, the equilibrium, as in Proposition 3 , is inefficient. Also, similar to the results in Sect. 3.2, the seller's revenue is higher while bidder's payoff is lower compared to the situation when no information is disclosed. Also, I find that disclosing both bids is still equivalent to disclosing only the winning bid.

Second, I assume that the valuations of each bidder are distributed across items as follows: $p_{10}=p_{01}=\frac{1}{2}-\epsilon$ and $p_{00}=p_{11}=\epsilon$ where $0 \leq \epsilon \leq \frac{1}{4}$. $\epsilon=0$ corresponds to the case analysed in Sect. 4 , while $\epsilon=\frac{1}{4}$ again implies 

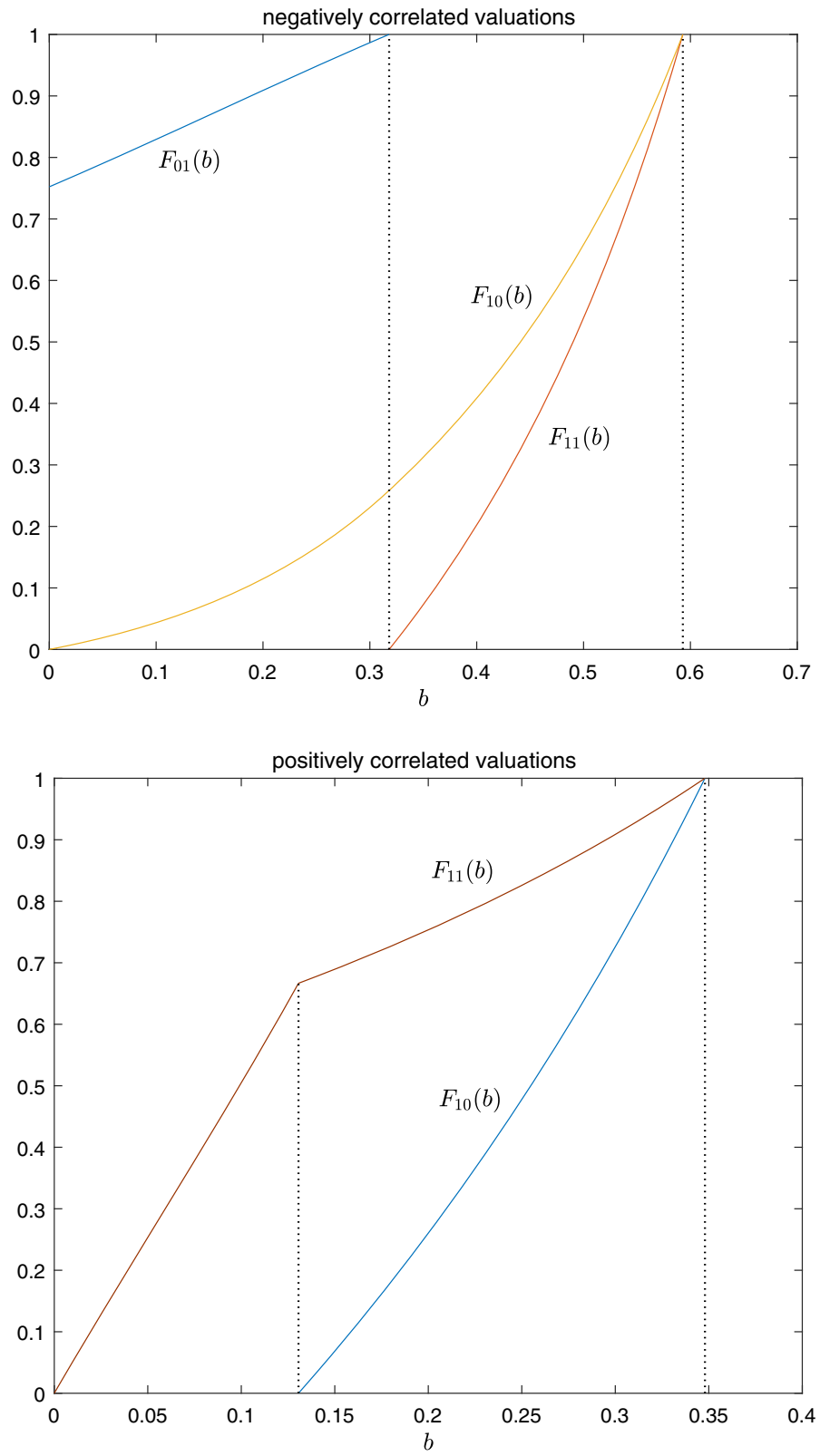

Fig. 4 Equilibrium strategies when $\epsilon=\frac{1}{8}$

that the valuations across items are independently distributed. When $0<\epsilon<\frac{1}{4}$, the valuations of the items are positively, but imperfectly correlated. If only the winning bid of the first auction is revealed, I find that in equilibrium, type $(1,1)$ randomizes on $[0, \bar{b}]$, type $(1,0)$ randomizes on $[\underline{b}, \bar{b}]$ interval where $\underline{b}>0$, while 
types $(0,0)$ and $(0,1)$ always bid 0 in the first auction. (See the bottom panel in Fig. 4.) In the limit, when $\epsilon$ goes to 0 , the equilibrium converges to the one given in Proposition 8 (with $p=\frac{1}{2}$ and $v=1$ ). Similar to the results in that proposition, the equilibrium is efficient for all $\epsilon \in\left(0, \frac{1}{4}\right)$. Also, as in Sect. 4.2, information disclosure leads to lower revenue for the seller and to higher payoffs for the bidders. $^{29}$

\subsection{Asymmetric distribution of valuations}

Here I generalize Proposition 3 to the case of asymmetric distribution of valuations. It is still assumed that $p_{00}=p_{11}=0$ for both bidders, but now $p_{01}=p^{1}$ for bidder 1 and $p_{01}=p^{2}$ for bidder 2 with $p^{1} \geq p^{2}$. I find that when only the winning bid of the first auction is revealed, both types of both bidders randomize over exactly the same interval $[0, \bar{b}]$ in equilibrium, provided that $p^{1} \geq p^{2} \geq \bar{b}$ holds. (When $p^{1}=p^{2} \geq \bar{b}$, this corresponds to the case when $p v \geq 1-e^{-v}$ and $v=1$ in Proposition 3.) Figure 5 illustrates the equilibrium strategies for $p^{1}=0.7$ and $p^{2}=0.5{ }^{30}$ Clearly, the equilibrium is still inefficient. I have also verified that compared to the case when no information is disclosed, the announcement of the winning bid always lowers the joint payoff of the bidders and raises the seller's revenue, which is consistent with the results in Sect. 3.2.

Because the bidders are asymmetric, the equivalence between disclosing only the winning bid and disclosing both bids does not hold anymore. Given the strategies of the first auction when only the winning bid is disclosed, one can show that $(1-$ $\left.p^{1}\right) f_{10}^{1}(b)>\left(1-p^{2}\right) f_{10}^{2}(b)$ holds for $b>0$. Further, if both $b_{w}$ and $b_{l}$ are disclosed and they are sufficiently close to each other, then $q_{w} \geq q_{l}$ will only hold if (1 $\left.p^{w}\right) f_{10}^{w}\left(b_{w}\right)>\left(1-p^{l}\right) f_{10}^{l}\left(b_{l}\right)$ holds. However, this will be violated if bidder 1 loses the first auction (that is, $l=1$ and $w=2$ ). Therefore, the equilibrium strategies when only the winning bid is disclosed, cease to form an equilibrium when both bids are disclosed. ${ }^{31}$

\footnotetext{
29 With perfectly positively correlated valuations, if only the winner's identity is announced, then at the start of the second auction, one of the bidders has private information about his valuation, while the other bidder has private information about his belief about the first bidder's valuation. This allowed to construct equilibrium strategies for the second auction and to show that the equilibrium outcome is the same as when the winning bid is announced. With imperfectly positively correlated valuations, if the bidders bid in the first auction as just described but only the winner's identity is announced, then at the start of the second auction, each bidder has private information both about his valuation and about his belief about the opponent's valuation. This make it hard to construct the equilibrium strategies for the second auction and suggests that the equivalence between the two disclosure policies does not hold anymore.

30 The superscript to the distribution function denotes the bidder.

31 It was problematic to identify the equilibria in the case of asymmetric bidders and positively correlated valuations. I constructed an equilibrium when both bids are announced. This equilibrium resembles the one given in Proposition 11. However, to construct it, I needed to adopt a very particular tie-breaking rule for the first auction, namely, the probability with which the item is assigned to, say, bidder 1 in the case of tie, is continuously changing in the values of $p^{1}$ and $p^{2}$.
} 


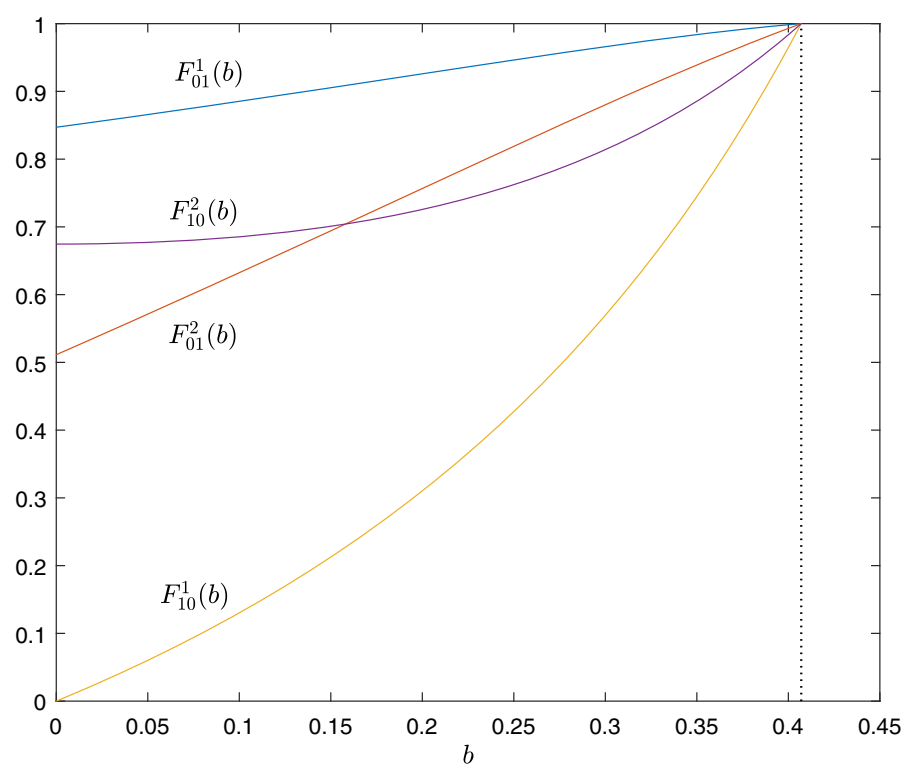

Fig. 5 Equilibrium strategies when $p^{1}=0.7$ and $p^{2}=0.5$

\subsection{More than two bidders}

Next, I consider the case of $n \geq 2$ bidders. Kannan (2012) has already analysed this case when the valuations are perfectly positively correlated and either the winning bid or all bids are announced. He finds that the equilibria are qualitatively the same when $n=2$ and when $n>2$. Specifically, as in Proposition 8, when only the winning bid is announced, type $(1,1)$ bidders bid above 0 with probability 1 and, hence, the equilibrium is efficient. And as in Proposition 11, when all bids are announced, type $(1,1)$ bidders bid 0 with a strictly positive probability and, hence, the equilibrium is inefficient.

I focus now on the case when the valuations are perfectly negatively correlated, but first I characterize the equilibrium behaviour and payoffs in the second auction. This characterization, though, does not depend on the sign of correlation between the valuations of the items. I start by introducing some additional terminology and notation. I will say that a bidder, depending on his valuation of the second item, is of type 0 or 1 in the second auction. Let $q_{i}(i=1, \ldots, n)$ denote the probability that bidder $i$ is of type 0 . Suppose (w.l.o.g.) that $q_{1} \leq q_{2} \leq \cdots \leq q_{n}$. I show that in equilibrium, bidder $i$ randomizes over the interval $\left[c_{i}, c_{n+1}\right]$ where $0 \leq c_{1}=c_{2} \leq$ $\cdots \leq c_{n} \leq c_{n+1}$. If $q_{i}=q_{i+1}$, then $c_{i}=c_{i+1}$ and bidders $i$ and $i+1$ have identical equilibrium strategies. The payoff of type 1 bidder is $\Pi_{j=2}^{n} q_{j}$, while that of type 0 is 0 .

Note that the payoff of type 1 bidder does not depend on the probability of the bidder who is the least likely to have the low valuation. Therefore, the disclosure rules that only differ how $q_{1}$ is updated based on the bidding in the first auction, must be 
equivalent. We have seen that with the negatively correlated valuations, $q_{i}$ is increasing in the bids of the first auction. Therefore, the bidder who submits the lowest bid in the first auction, is the least likely to have the low valuation for the second item. Therefore, the disclosure rule when all bids are announced, must be equivalent to the disclosure rule when all but the lowest bid are announced. A similar argument holds when the valuations are positively correlated, except that then $q_{i}$ is decreasing in the bids of the first auction.

Furthermore, even if two disclosure rules lead to different updated values of $q_{i}$ for $i=2, \ldots, n$ given the bids of the first auction, these disclosure rules can still be equivalent. Specifically, I find that when the valuations are negatively correlated and there are at least 3 bidders, the equilibrium outcomes and equilibrium payoffs are the same when no information about the first auction is announced and when only the winning bid is announced. In the equilibrium, type $(0,1)$ bidders bid 0 and type $(1,0)$ bidders bid strictly above 0 in the first auction and it is the other way around in the second auction. As a result, the equilibrium outcome is efficient.

Note that it is different in the two bidder case. There, a type $(0,1)$ bidder bids above 0 with a strictly positive probability in the first auction and the outcome is inefficient. If in the two bidder case, type $(0,1)$ bidders were expected to bid 0 for sure as in the $n>2$ case, then a type $(0,1)$ bidder would have incentives to deviate by submitting a small but positive bid. This way he can secure a payoff of almost 1 in the second auction: either he loses the first auction and learns that his opponent will bid 0 in the second auction, or he wins and makes the opponent to believe that he will bid 0 in the second auction. With 3 or more bidders, such a deviation has only a marginal effect on the payoff in the second auction as there still remains uncertainty about the types of other bidders. And this marginal change in the payoff from the second auction is outweighed by the cost of winning the first auction with a bid above one's valuation. ${ }^{32}$

\subsection{Other disclosure rules}

I have studied simple, "natural" disclosure rules, but the seller could also adopt a more sophisticated disclosure rule. I now consider an example of such more sophisticated rule and show that it outperforms the simple rules in the case of negatively correlated valuations. The disclosure rule that I consider is as follows. The seller announces that the winning bid of the first auction is $b_{w}$ if $b_{w}<k$ where $k$ is some threshold value, but she announces that the losing bid is $b_{l}$ if $b_{w} \geq k$. To emphasize, the seller does not just announce a number. She also reveals whether she announces the winning or losing bid. Said differently, she reveals whether or not the bids $\left(b_{w}, b_{l}\right)$ belong to the set $B=\left\{\left(b_{w}, b_{l}\right) \mid b_{w}<k\right\}$.

The bidding strategies of the first auction are similar to the ones in Proposition 5 when only the losing bid is disclosed. In that proposition, type $(0,1)$ randomizes on an interval $[1-p, \underline{b}]$, while type $(1,0)$ randomizes on an adjacent interval $[\underline{b}, \bar{b}]$. By choosing $k>1-p, \underline{b}$ is now pushed to the right. Although type $(0,1)$ still expects

\footnotetext{
32 Although I have not derive the equilibrium strategies when all bids of the first auction are announced, I have verified that with 3 bidders, it cannot be an equilibrium where type $(0,1)$ bidders bid 0 and type $(1,0)$ bidders bid strictly above 0 in the first auction.
} 
the same payoff as in the case when only the losing bid is disclosed, type $(1,0)$ must now bid more aggressively because of higher $\underline{b}$ and, as a result, expects smaller payoff. This, in turn, means higher revenue for the seller.

Open Access This article is licensed under a Creative Commons Attribution 4.0 International License, which permits use, sharing, adaptation, distribution and reproduction in any medium or format, as long as you give appropriate credit to the original author(s) and the source, provide a link to the Creative Commons licence, and indicate if changes were made. The images or other third party material in this article are included in the article's Creative Commons licence, unless indicated otherwise in a credit line to the material. If material is not included in the article's Creative Commons licence and your intended use is not permitted by statutory regulation or exceeds the permitted use, you will need to obtain permission directly from the copyright holder. To view a copy of this licence, visit http://creativecommons.org/licenses/by/4.0/.

\section{A Proofs}

Proof of Proposition 3 First of all, one can verify that the bidders' beliefs at the start of the second auction, as described by the first expression for each $q_{i}$ for $i=w, l$, are consistent with the strategies of the first auction. (Although, bidder $l$ additionally knows his losing bid, it does not provide any additional information about the opponent's type.) Further, the density functions for $0 \leq b \leq \bar{b}$ are given by

$$
\begin{aligned}
& f_{01}(b)=F_{01}(0) \frac{v+\ln (1-b)}{v(1-b)^{2}} \\
& f_{10}(b)=F_{01}(0) \frac{p}{1-p} \frac{-\ln (1-b)}{v(1-b)^{2}} .
\end{aligned}
$$

One can verify that $f_{01}(b)>0$ and $f_{10}(b)>0$ for all $0<b<\bar{b}$ as required. Using these expressions for $f_{01}(b)$ and $f_{10}(b)$, one can simplify the expressions for $q_{w}$ and $q_{l}$ when $0 \leq b_{w} \leq \bar{b}$ :

$$
\begin{aligned}
q_{w} & =-\frac{\ln \left(1-b_{w}\right)}{v} \\
q_{l} & =-\frac{b_{w}+\ln \left(1-b_{w}\right)}{v}
\end{aligned}
$$

Hence, $q_{l} \leq q_{w}$ clearly holds, therefore one can apply Lemma 1. Finally, one can also verify that $f_{01}\left(1-e^{-v}\right)=0$. This is important because it ensures that there is no discrete jump in $q_{w}$ at $b_{w}=\bar{b}=1-e^{-v}$ when $p v<1-e^{-v}$. Consequently, $q_{w}=1$ for all $b \geq \bar{b}$ when $p v<1-e^{-v}$.

Next, we consider the bidders' behaviour in the first auction. Consider type $(0,1)$. If he bids in the interval $[0, \bar{b}]$, his joint payoff from both auctions is ${ }^{33}$

$$
\pi_{01}=\left(p F_{01}(b)+(1-p) F_{10}(b)\right) \times(-b)
$$

33 If $b=0$, we need to consider ties. However, the first two terms in the payoff expression will be zero for any tie-breaking rule. 


$$
\begin{aligned}
& +\left(p F_{01}(b)+(1-p) F_{10}(b)\right) \times \frac{(1-p) f_{10}(b)}{p f_{01}(b)+(1-p) f_{10}(b)} \times v \\
& +\left(p\left(1-F_{01}(b)\right)+(1-p)\left(1-F_{10}(b)\right)\right) \\
& \times \int_{b}^{b_{10}} v \times \frac{(1-p) f_{10}(s)}{p f_{01}(s)+(1-p) f_{10}(s)} \\
& \times \frac{p f_{01}(s)+(1-p) f_{10}(s)}{p\left(1-F_{01}(b)\right)+(1-p)\left(1-F_{10}(b)\right)} d s,
\end{aligned}
$$

where the first term is the bidder's payoff from the first auction, the second and third terms are his payoff from the second auction if he has, respectively, won and lost the first auction, ${ }^{34}$ and $\bar{b}_{10}=\bar{b}$ if $p v \geq 1-e^{-v}$, and $\bar{b}_{10}=1-\frac{p v}{e^{v}-1}$ otherwise. If we simplify the above expression, we obtain that

$$
\begin{aligned}
\pi_{01} & =-\left(p F_{01}(b)+(1-p) F_{10}(b)\right)(b+\ln (1-b))+(1-p)\left(1-F_{10}(b)\right) v \\
& =(1-p) v,
\end{aligned}
$$

where the last equality follows from substituting the expressions for $F_{01}(b)$ and $F_{10}(b)$.

If he bids above $\bar{b}$, the expression for the payoff depends on whether $p v<1-e^{-v}$ or $p v \geq 1-e^{-v}$. If $p v<1-e^{-v}$, then $q_{w}=1$ and for $b \in\left(\bar{b}, 1-\frac{p v}{e^{v}-1}\right]$, the payoff is

$$
\left(p+(1-p) F_{10}(b)\right)(v-b)+(1-p)\left(1-F_{10}(b)\right) v=v\left(1-\frac{p}{e^{v}-1} \frac{b}{1-b}\right),
$$

where I have used (4). Because $b>\bar{b}=1-e^{-v}$, this payoff is less than $(1-p) v$. For $b>1-\frac{p v}{e^{v}-1}$, the payoff is

$$
v-b<v-1+\frac{p v}{e^{v}-1}<v-1+e^{-v}<(1-p) v .
$$

If $p v \geq 1-e^{-v}$, then for $b>\bar{b}$ the payoff is

$$
\begin{array}{r}
-b+\frac{(1-p) f_{10}(\bar{b})}{p f_{01}(\bar{b})+(1-p) f_{10}(\bar{b})} v=-b-\ln (1-\bar{b}) \\
<-\bar{b}-\ln (1-\bar{b})=(1-p) v
\end{array}
$$

It follows that the payoff of type $(0,1)$ is maximized for $b \in[0, \bar{b}]$. Thus, it is indeed optimal for this type to randomize on this interval.

Consider type $(1,0)$. If he bids in the interval $(0, \bar{b}]$, his expected payoff is

$$
\pi_{10}=\left(p F_{01}(b)+(1-p) F_{10}(b)\right)(1-b)=p F_{01}(0),
$$

\footnotetext{
34 Recall that the payoff of type $(0,1)$ from the second auction is given by $q_{w} v$, where $q_{w}$ depends on the winning bid of the first auction.
} 
where the last equality follows from substituting the expressions for $F_{01}(b)$ and $F_{10}(b)$. If $p v<1-e^{-v}$, then for any $b \in\left(\bar{b}, 1-\frac{p v}{e^{v}-1}\right]$, the expected payoff is

$$
\left(p+(1-p) F_{10}(b)\right)(1-b)=p F_{01}(0),
$$

where I have used (4). If he bids 0 , then his expected payoff is $\frac{1}{2} p F_{01}(0)$, given the tiebreaking rule. If he bids above $\bar{b}_{10}$, his expected payoff is $1-b<1-\bar{b}_{10}=p F_{01}(0)$. Thus, this type is indifferent between all bids $\left(0, \bar{b}_{10}\right]$ and, hence, is indeed willing to randomize according to $F_{10}(b)$.

Proof of Proposition 5 First, the bidders' beliefs at the start of the second auction, as described by $q_{w}$ and $q_{l}$, are derived from the strategies of the first auction using Bayes' rule whenever possible. Second, because $q_{l} \leq q_{w}$ holds, one can apply Lemma 1 to the second auction.

We now turn to the first auction. First, however, note that given the specified strategies, ties will arise with zero probability. (In particular, $\lim _{b \rightarrow((1-p) v)^{+}} F_{01}(b)=0$.) Consider type $(0,1)$. If he bids in the interval $\left[(1-p) v,-\ln (1-p) \frac{1-p}{p} v\right]$, his expected payoff from both auctions is

$$
\begin{aligned}
\pi_{01}= & p F_{01}(b)(-b)+\left(1-p+p\left(1-F_{01}(b)\right)\right) \frac{1-p}{1-p+p\left(1-F_{01}(b)\right)} v \\
& +p F_{01}(b) \int_{(1-p) v}^{b} \frac{1-p}{1-p+p\left(1-F_{01}(s)\right)} v \frac{f_{01}(s)}{F_{01}(b)} d s \\
= & p F_{01}(b)(-b)+(1-p) v-(1-p) v \int_{(1-p) v}^{b} \frac{d\left(1-p F_{01}(s)\right)}{1-p F_{01}(s)} \\
= & p F_{01}(b)(-b)+(1-p) v-(1-p) \ln \left(1-p F_{01}(b)\right) v \\
= & (1-p) v,
\end{aligned}
$$

where the last equality follows from the definition of $F_{01}(b)$ in $(5)$. Thus, type $(0,1)$ is indifferent between all bids in the interval $\left[(1-p) v,-\ln (1-p) \frac{1-p}{p} v\right]$. If this type bids below $(1-p) v$, he expects the same payoff as when bidding $(1-p) v$. If he bids above $-\ln (1-p) \frac{1-p}{p} v$, he expects a payoff of

$$
\begin{aligned}
& \left(p+(1-p) F_{10}(b)\right)(-b)+(1-p) v-(1-p) \ln (1-p) v \\
& =(p+(1-p) \ln (1-p) v) \frac{-b}{1-b}+(1-p)(1-\ln (1-p)) v
\end{aligned}
$$

which is decreasing in $b$ because $p+(1-p) \ln (1-p) v>0$. It follows that it is indeed optimal for type $(0,1)$ to randomize according to $F_{01}(b)$ in the first auction. 
Consider type $(1,0)$. If he bids in the interval $\left[-\ln (1-p) \frac{1-p}{p} v,(1-p)\right.$ $(1-\ln (1-p) v)]$, his expected payoff is

$$
\begin{aligned}
\pi_{10} & =\left(p+(1-p) F_{10}(b)\right)(1-b) \\
& =\left(p+(1-p) \frac{p}{1-p} \frac{b+\ln (1-p) \frac{1-p}{p} v}{1-b}\right)(1-b) \\
& =p+(1-p) \ln (1-p) v .
\end{aligned}
$$

If he bids above $(1-p)(1-\ln (1-p) v)$, he clearly expects less. If he bids below $-\ln (1-p) \frac{1-p}{p}$, his payoff is

$$
p F_{01}(b)(1-b)=p F_{01}(b)+(1-p) \ln \left(1-p F_{01}(b)\right) v
$$

where I have used (5). One can verify that the last expression is increasing in $b$ for $v \leq 1$. Thus, it follows that it is indeed optimal for type $(1,0)$ to randomize according to $F_{10}(b)$ in the first auction.

Proof of Proposition 6 We solve for the equilibrium strategies backwards, starting with the second auction. Given the specified strategies, it is clear that type $(1,0)$ finds it optimal to bid 0 for any beliefs about the type of the opponent. It remains to check the optimality of type $(0,1)$ strategies. At the beginning of second auction, bidder $w$ of type $(0,1)$ believes that the opponent is of type $(1,0)$ with probability $q_{l}=0$. His expected payoff at the start of the second auction is

$$
H_{l}(c)(v-c)=q_{w} v
$$

for all $c \in\left[0,\left(1-q_{w}\right) v\right]$ and it is $v-c<q_{w} v$ for $c>\left(1-q_{w}\right) v$. Thus, this bidder is willing to bid $c_{w}$ as long as $c_{w} \leq\left(1-q_{w}\right) v=\frac{p\left(1-F_{01}\left(b_{l}\right)\right)}{1-p+p\left(1-F_{01}\left(b_{l}\right)\right)} v=c_{l}$ where the first equality follows from (6) and the second equality is simply a definition $\left(c_{l} \equiv c\left(b_{l}\right)\right)$. Because $c(b)$ is decreasing in $b$ and $b_{w} \geq b_{l}$, it is indeed true that $c_{w} \leq c_{l}$.

On the other hand, bidder $l$ of type $(0,1)$ believes that the opponent is of type $(1,0)$ with probability $q_{w}$, which is given in (6). Furthermore, conditional on his first auction losing bid $b_{l}$ and conditional on the opponent being of type $(0,1)$, bidder $l$ believes that the opponent's bid in the first auction exceeds $b$ with probability $\frac{1-F_{01}(b)}{1-F_{01}\left(b_{l}\right)}$, or equivalently that the opponent's bid in the second auction is less than $c$ with probability $\frac{H_{w}(c)}{H_{w}\left(c_{l}\right)}$ where $c_{l}=\left(1-q_{w}\right) v$. Therefore, the expected payoff of this type at the start of the second auction is

$$
\left(q_{w}+\left(1-q_{w}\right) \frac{H_{w}(c)}{H_{w}\left(c_{l}\right)}\right)(v-c) .
$$

Substituting the expressions for $q_{w}$ and $H_{w}(c)$, we obtain that

$$
\left(\frac{1-p+p\left(1-F_{01}\left(b_{l}\right)\right) \frac{H_{w}(c)}{H_{w}\left(c_{l}\right)}}{1-p+p\left(1-F_{01}\left(b_{l}\right)\right)}\right)(v-c)=\frac{1-p}{1-p+p\left(1-F_{01}\left(b_{l}\right)\right)} v=q_{w} v
$$


for all $c \in\left[0,\left(1-q_{w}\right) v\right]$. If he bids above $\left(1-q_{w}\right) v$, his payoff is clearly less than $q_{w} v$. Because this type of bidder is indifferent between all bids in the interval $\left[0,\left(1-q_{w}\right) v\right]$, it is indeed optimal to randomize according to $H_{l}(c)$.

Hence, type $(0,1)$ expects a payoff of $q_{w} v$, which depends on $b_{l}$, in the second auction whether or not he has won the first auction. It immediately follows that the equilibrium strategies in the first auction are exactly the same as the ones in Proposition 5.

Proof of Proposition 8 First, the beliefs at the start of the second auction round are consistent with the first round strategies. Second, $q_{w} \leq q_{l}$ holds. Third, the lowest bid that type $(1,1)$ submits in the first auction is 0 , therefore it is indeed optimal for type $(0,0)$ to bid 0 in that auction.

The expected payoff of type $(1,1)$ bidder at the start of the first auction is

$$
\begin{aligned}
& (p+(1-p) F(b))(1-b)+(p+(1-p) F(b)) \frac{p v}{p+(1-p) F(b)} \\
& \quad+(1-p)(1-F(b)) \int_{b}^{\bar{b}} \frac{p v}{p+(1-p) F(s)} \frac{f(s)}{1-F(b)} d s
\end{aligned}
$$

where the first term is the bidder's payoff from the first auction, the second and third terms are his payoff from the second auction if he has, respectively, won and lost the first auction, ${ }^{35}$ and $\bar{b} \equiv 1-p+v p \ln p$. After simplification, we obtain

$$
(p+(1-p) F(b))(1-b)+v p-v p \ln (p+(1-p) F(b)) \text {. }
$$

Substituting the expression for $F(b)$ from (10) implies that the payoff is equal to $p(1+v(1-\ln p))$ for all $b \in[0, \bar{b}]$. Clearly, the payoff is strictly lower if the bidder bids above $\bar{b}$. Hence, a bidder with the valuation profile $(1,1)$ is indeed willing to randomize according to $F(b)$ in the first auction. This completes the proof.

Proof of Proposition 9 It is trivial to see that it is optimal for type $(0,0)$ to bid 0 in both auctions, given the strategy of the opponent. Consider type $(1,1)$. We start with the second auction. Prior to the second auction, bidder $l$ (of type $(1,1)$ ) believes that the opponent's type is $(0,0)$ with probability $q_{w}=0$, while bidder $w$ believes that his opponent's type is $(0,0)$ with probability $q_{l}$, which is given in (11).

The expected payoff of bidder $l$, conditional on $c_{w}$, is

$$
H_{w}(c)(v-c)=v-c_{w}
$$

for all $c \leq c_{w}$ and it is $v-c<v-c_{w}$ for any $c>c_{w}$. Thus, even if bidder $l$ does not know what $c_{w}$ is, he knows that his payoff is maximized at $c=c_{l}$ (because $c_{l} \leq c_{w}$ ).

The expected payoff of bidder $w$ is

$$
\left(q_{l}+\left(1-q_{l}\right) \frac{H_{l}(c)}{H_{l}\left(c_{w}\right)}\right)(v-c) .
$$

\footnotetext{
35 Recall that the payoff of type $(1,1)$ from the second auction is given by $q_{l} v$, where $q_{l}$ depends on the winning bid of the first auction according to (11).
} 
Substituting the expressions for $q_{l}$ and $H_{l}(c)$ in the payoff function and noting that $F\left(b_{w}\right)=H_{l}\left(c_{w}\right)$, we obtain that the payoff is $v-c_{w}$ for $c \leq c_{w}$, but the payoff is $v-c<v-c_{w}$ for $c>c_{w}$. Thus, bidder $w$ is indeed willing to randomize according to $H_{w}(c)$.

Note that from (11) and (13), $c_{w}=\left(1-q_{l}\right) v$. It follows that type $(1,1)$ expects a payoff of $q_{l} v$ in the second auction whether he wins or loses the first auction. From this and the fact that the relationship between $q_{l}$ and $b_{w}$ is the same as when the winning bid is disclosed, it immediately follows that the equilibrium strategy of type $(1,1)$ in the first auction is exactly the same as the one given in Proposition 8. This completes the proof.

Proof of Proposition 10 The beliefs prior to the second auction are indeed consistent with the bidding strategies of the first auction; $q_{w} \leq q_{l}$ holds; type $(0,0)$ bidder finds it optimal to bid 0 in the first auction.

Consider type $(1,1)$ in the first auction when $p<\frac{2 v}{1+2 v}$. If he bids $b>0$, then his expected payoff is

$$
\begin{aligned}
& (p+(1-p) F(b))(1-b)+(p+(1-p) F(0)) \times \frac{p v}{p+(1-p) F(0)} \\
& \quad+(1-p)(1-F(0)) \times 0 .
\end{aligned}
$$

If he bids $b=0$, then the payoff is

$$
(p+(1-p) F(0)) \times \frac{1}{2} \times 1+\frac{p v}{p+(1-p) F(0)} .
$$

Substituting the expression for $F(b)$, it follows that the expected payoff both from bidding $b>0$ and from bidding $b=0$ is the same and equal to $\sqrt{p v(2+p v)}$. Obviously, the bidder has no incentives to bid above $\bar{b}=1+p v-\sqrt{p v(2+p v)}$ as it is dominated by bidding $\bar{b}$.

When $\frac{2 v}{1+2 v} \leq p<1$, type $(1,1)$ expects

$$
(p+(1-p) F(b))(1-b)+p \times v+(1-p) \times 0=p(1+v)
$$

for $0<b \leq 1-p$. If he bids $b=0$, his expected payoff is

$$
p \times \frac{1}{2} \times 1+v
$$

which is (weakly) less than $p(1+v)$ when $\frac{2 v}{1+2 v} \leq p<1$. He has no incentives to bid above $1-p$ either. We conclude that $F$ is indeed the equilibrium strategy of type $(1,1)$ bidder in the first auction. 


\section{B Calculating $\Delta_{n}$}

Here, using the results of Sect. 3.2, I calculate explicitly

$$
\Delta_{n}=p\left(\int_{0}^{\bar{b}_{10}} F_{01}(b) f_{10}(b) d b-F_{01}(0)\right)+(1-p) \ln (1-p) v .
$$

When $p v \geq 1-e^{-v}$, the integral in the above expression is given by

$$
-\frac{(1-\bar{b})^{2}}{p(1-p) v^{2}} \int_{0}^{\bar{b}} \frac{(v+b+\ln (1-b)) \ln (1-b)}{(1-b)^{3}} d b=\frac{\bar{b}^{2}+2 p(1-p) v}{4 p(1-p) v},
$$

where I have used the relationship $\ln (1-\bar{b})=-\bar{b}-(1-p) v$. Hence,

$$
\Delta_{n}=\frac{\bar{b}^{2}+2 p(1-p) v}{4(1-p) v}-(1-\bar{b})+(1-p) \ln (1-p) v
$$

and $\bar{b}$ is given by $v(1-p)+\bar{b}+\ln (1-\bar{b})=0$.

When $p v<1-e^{-v}$, the integral is given by

$$
\begin{aligned}
1- & F_{10}\left(1-e^{-v}\right)+\int_{0}^{1-e^{-v}} F_{01}(b) f_{10}(b) d b \\
= & 1+\frac{p}{1-p} \frac{1-v-e^{-v}}{1-e^{-v}}-\frac{p}{1-p} \frac{1}{\left(e^{v}-1\right)^{2}} \\
& \int_{0}^{1-e^{-v}} \frac{(v+b+\ln (1-b)) \ln (1-b)}{(1-b)^{3}} d b \\
= & 1+\frac{p}{1-p} \frac{e^{v}-v e^{v}-1}{e^{v}-1}-\frac{1}{4} \frac{p}{1-p} \frac{-v-4 e^{v}+2 e^{2 v}-3 v e^{2 v}+4 v e^{v}+2}{\left(e^{v}-1\right)^{2}} .
\end{aligned}
$$

Hence,

$$
\Delta_{n}=p\left(1+\frac{3 p v-4 v-2 p+2 p e^{v}-p v e^{v}}{4\left(e^{v}-1\right)(1-p)}\right)+(1-p) \ln (1-p) v .
$$

\section{References}

Azacis H (2019) Information disclosure by a seller in sequential first-price auctions. Cardiff Economics Working Paper E2017/2, Cardiff Business School

Azacis H, Vida P (2012) Collusive communication schemes in a first-price auction. Cardiff Economics Working Paper E2012/11, Cardiff Business School

Bergemann D, Hörner J (2018) Should first-price auctions be transparent? Am Econ J Microecon 10(3): 177218

Cason TN, Kannan KN, Siebert R (2011) An experimental study of information revelation policies in sequential auctions. Manag Sci 57(4):667-688 
Ding W, Jeitschko TD, Wolfstetter EG (2010) Signal jamming in a sequential auction. Econ Lett 108:58-61 Esponda I (2008) Information feedback in first price auctions. RAND J Econ 39(2):491-508

Fan C, Jun BH, Wolfstetter EG (2016) Optimal bid disclosure in patent license auctions under alternative modes of competition. Int J Ind Org 47:1-32

Février P (2003) He who must not be named. Rev Econ Des 8:99-119

Giovannoni F, Makris M (2014) Reputational bidding. Int Econ Rev 55(3):693-710

Jehiel P (2011) Manipulative auction design. Theor Econ 6(2):185-217

Jeitschko TD (1998) Learning in sequential auctions. South Econ J 65(1):98-112

Kannan KN (2012) Effects of information revelation policies under cost uncertainty. Inf Syst Res 23(1):7592

Lebrun B (2010) First-price auctions with resale and with outcomes robust to bid disclosure. RAND J Econ 41(1):165-178

Maskin ES, Riley JG (1985) Auction theory with private values. Am Econ Rev 75(2):150-155

Thomas CJ (2010) Information revelation and buyer profits in repeated procurement competition. J Ind Econ 58(1):79-105

Tu Z (2005) Three essays on auctions. Ph.D. thesis, University of Pittsburgh

Yao Z, Xiao Z (2013) A note on sequential auctions with multi-unit demand. Math Soc Sci 66(3):276-281

Publisher's Note Springer Nature remains neutral with regard to jurisdictional claims in published maps and institutional affiliations. 